\title{
Finite element approximation of turbulent thermally coupled incompressible flows with numerical sub-grid scale modeling
}

\author{
Ramon Codina, Javier Principe and Matías Ávila \\ Universitat Politècnica de Catalunya, Jordi Girona 1-3, Edifici C1, 08034 Barcelona, Spain. \\ * ramon.codina@upc.edu, principe@cimne.upc.edu, matiasavila@hotmail.com
}

\section{Contents}

1 Introduction $\quad 2$

2 Finite element approximation $\quad 3$

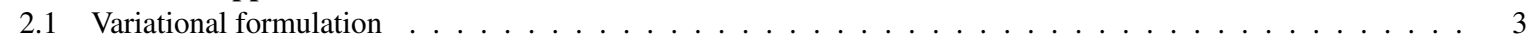

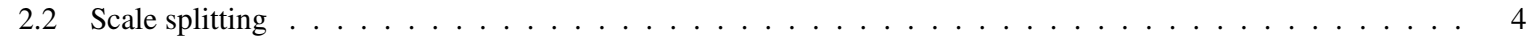

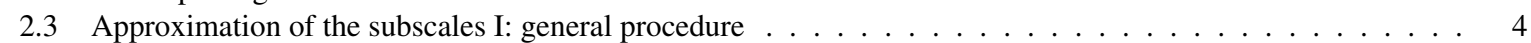

2.4 Approximation of the subscales II: application to thermally coupled flows $\ldots \ldots \ldots \ldots \ldots$

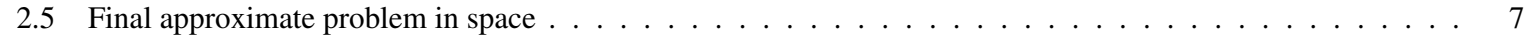

2.6 Main properties of the formulation $\ldots \ldots \ldots \ldots \ldots \ldots \ldots \ldots$

3 Conservation properties and energy transfer mechanisms $\quad 9$

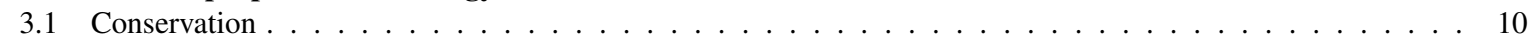

3.1.1 Conservation of linear momentum and heat . . . . . . . . . . . . . . . . . . . . . . 10

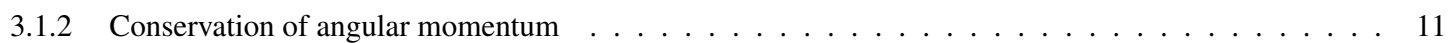

3.1.3 Conservation of kinetic energy and heat energy . . . . . . . . . . . . . . . . . . . 12

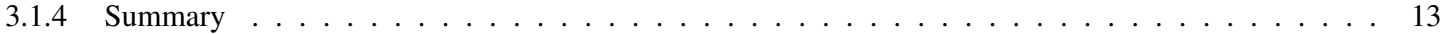

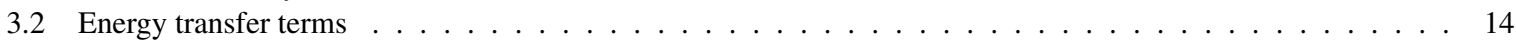

3.3 Numerical dissipation . . . . . . . . . . . . . . . . . . . . . . . . 15

4 Numerical examples $\quad 18$

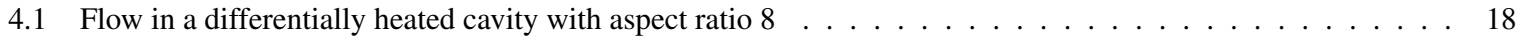

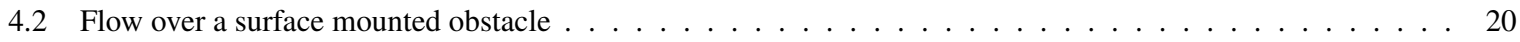

5 Conclusions $r$ 


\begin{abstract}
The objective of this work is to describe a variational multiscale finite element approximation for the incompressible Navier-Stokes equations using the Boussinesq approximation to model thermal coupling. The main feature of the formulation in contrast to other stabilized methods is that we consider the subscales as transient and orthogonal to the finite element space. These subscales are solution of a differential equation in time that needs to be integrated. Likewise, we keep the effect of the subscales both in the nonlinear convective terms of the momentum and temperature equations and, if required, in the thermal coupling term of the momentum equation. This strategy allows us to approach the problem of dealing with thermal turbulence from a strictly numerical point of view and discuss important issues, such as the relationship between the turbulent mechanical dissipation and the turbulent thermal dissipation.
\end{abstract}

Key Words: Thermally coupled flows, variational scale splitting, dynamic subscales

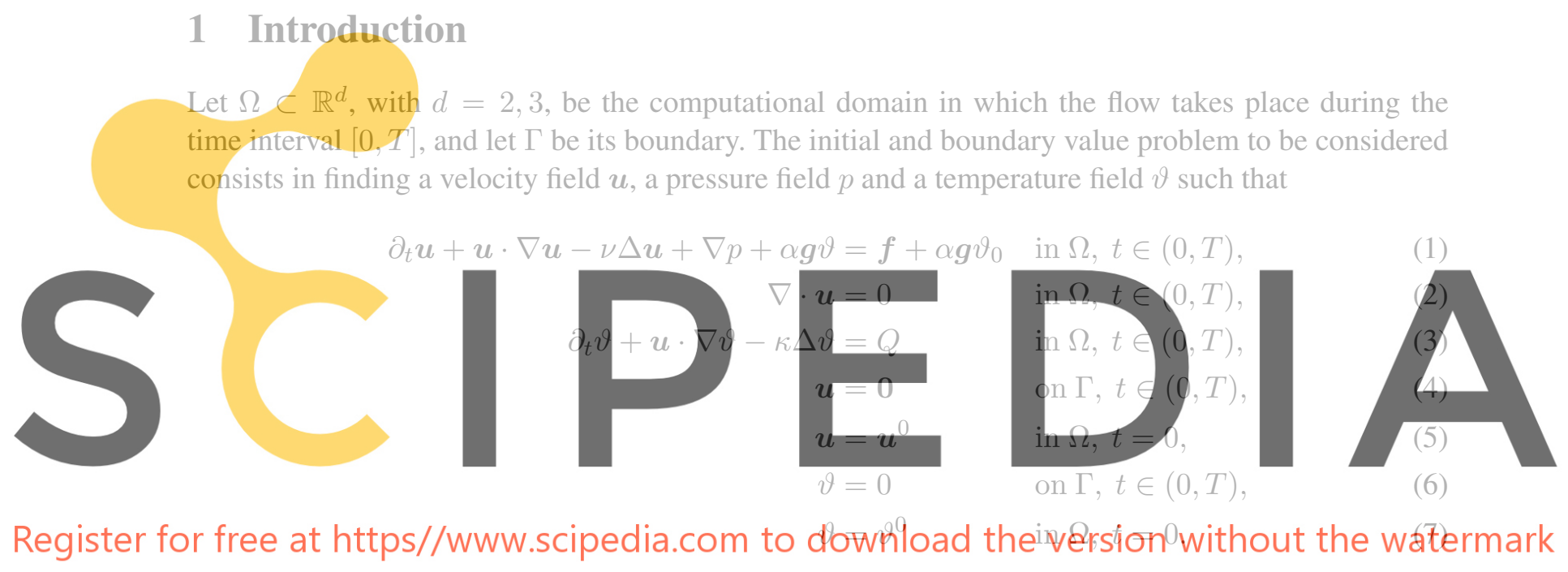

In these equations, $\nu$ is the kinematic viscosity, $\kappa$ the thermal diffusivity, $\alpha$ the thermal expansion coefficient, $\boldsymbol{f}$ the external body forces, $\vartheta_{0}$ the reference temperature, $\boldsymbol{g}$ the gravity acceleration vector, $Q$ the heat source and $\boldsymbol{u}^{0}$ and $\vartheta^{0}$ the initial conditions for velocity and temperature, respectively.

The literature on the finite element approximation of problem (1)-(7) is vast (see for example the introductory text [24]). The spatial discretization suffers from the well known problems of compatibility conditions between the velocity and pressure finite element spaces as well as the instabilities due to convection dominated flows, in this case both in the momentum equation (1) and the heat equation (3).

Apart from numerical difficulties, the physics modeled by (1)-(7) is extremely complex. In particular, turbulence should be in principle modeled by this system of equations. Since it is commonly accepted that turbulent scales cannot be captured in most applications, turbulence models of different complexity have been developed (see $[28,14]$ for background).

In recent years, the idea of using numerical techniques able to cope with the potential instabilities and to model turbulence at the same time has gained adepts, in particular within the variational multiscale concept introduced in $[16,17]$. The original motivation of this type of formulations was to justify the so called stabilized finite element methods. The possibility to model turbulence was remarked in [6] by contrast with the option adopted in [18] to add a large-eddy-simulation (LES) type model for the subgrid scales (see Remark 6 in [6] and, for background on LES models, [21]). In [2] the possibility to model turbulence using only numerical ingredients within the variational multiscale context is fully and successfully exploited. The role of numerical stabilization terms to model turbulence had also been 
envisaged in $[12,15]$, for example. For similar ideas using other numerical formulations, see $[4,25]$ and references therein.

The purpose of this paper is to give an overview of the finite element model proposed in $[6,11$, $13,23,9]$ and to present a complete description of its main properties, including the latest results obtained. It turns out that these properties support our view of modeling thermal turbulence from a strictly numerical point of view. Even though we restrict ourselves to the Boussinesq model described above, similar ideas can be applied to more complex thermally coupled flow models, as the low Mach number model whose finite element approximation is described in [22]. Our formulation is reviewed in Section 2, where some additional developments concerning the modeling of the subgrid scales are included. Section 3 contains a thorough discussion about the conservation properties of the scheme and the energy transfer mechanisms, which are relevant to model turbulent flows. In particular, we show how the numerical model we propose allows for a natural scale splitting in the energy balance, both in the mechanical and in the thermal problems. Likewise, the relationship with the dissipation introduced by classical LES models is described. Section 4 presents the results of two numerical examples, one of them showing the transition to turbulence as the Rayleigh number increases and the other showing the results of a numerical simulation of a turbulent flow. Conclusions and final remarks close the paper in Section 5 .

\section{Finite element approximation}
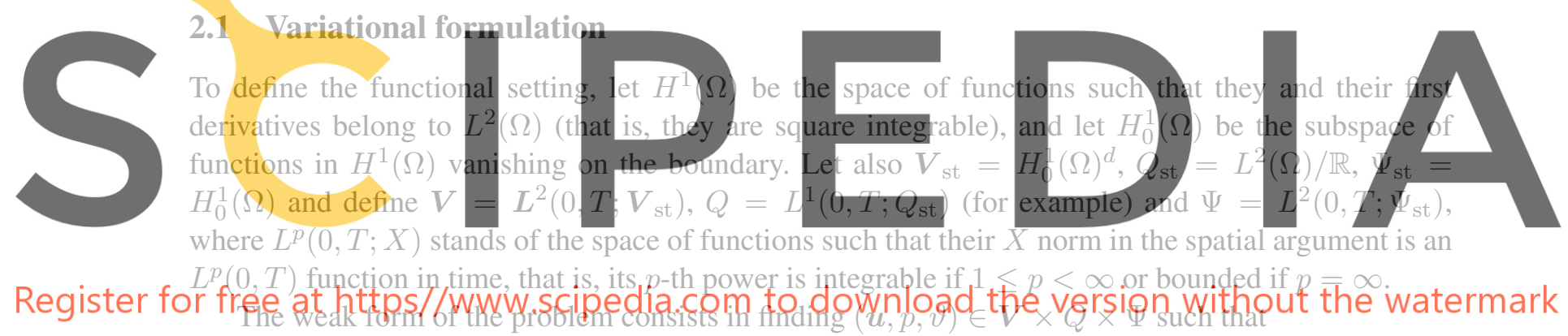

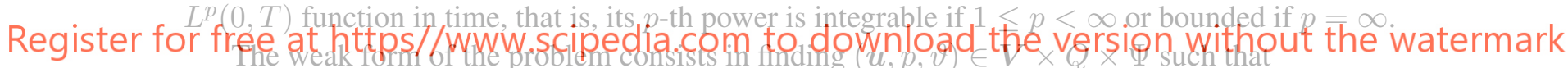

$$
\begin{aligned}
\left(\partial_{t} u, v\right)+\langle u \cdot \nabla u, v\rangle+\nu(\nabla u, \nabla v)-(p, \nabla \cdot v)+\alpha(g \vartheta, v) & =\langle f, v\rangle+\alpha\left(g \vartheta_{0}, v\right), \\
(q, \nabla \cdot \boldsymbol{u}) & =0, \\
\left(\partial_{t} \vartheta, \psi\right)+\langle\boldsymbol{u} \cdot \nabla \vartheta, \psi\rangle+\kappa(\nabla \vartheta, \nabla \psi) & =\langle Q, \psi\rangle,
\end{aligned}
$$

for all $(\boldsymbol{v}, q, \psi) \in \boldsymbol{V}_{\text {st }} \times Q_{\text {st }} \times \Psi_{\text {st }}$, where $(\cdot, \cdot)$ denotes the $L^{2}(\Omega)$ inner product and $\langle f, g\rangle:=\int_{\Omega} f g$ whenever functions $f$ and $g$ are such that the integral is well defined.

The dimensionless numbers relevant in this problem are

$$
\begin{aligned}
\operatorname{Re} & :=\frac{L U}{\nu}, & & \text { Reynolds number, } \\
\operatorname{Pe} & :=\frac{L U}{\kappa}, & & \text { Péclet number, } \\
\operatorname{Pr}: & =\frac{\nu}{\kappa} & & \text { Prandtl number, } \\
\operatorname{Ra} & :=\frac{\alpha|g| L^{3} \delta \vartheta}{\nu \kappa} & & \text { Rayleigh number. }
\end{aligned}
$$

where $L$ is a characteristic length of the problem, $U$ a characteristic velocity and $\delta \vartheta$ a characteristic temperature difference, usually computed from temperature boundary values when these are not zero. When $U$ cannot be determined by the boundary conditions, for example because zero velocities are prescribed, $U=\nu / L$ can be taken, which corresponds to choose $\operatorname{Re}=1$ and gives $\operatorname{Pe}=\operatorname{Pr}$. 


\subsection{Scale splitting}

Let us consider a finite element partition $\{K\}$ with $n_{\mathrm{e}}$ elements of the computational domain $\Omega$, from which we can construct finite element spaces for the velocity, pressure and temperature in the usual manner. We will assume that they are all built from continuous piecewise polynomials of the same degree $k$.

The basic idea of the multiscale approach we will follow [17] is to split the continuous unknowns as

$$
\begin{aligned}
\boldsymbol{u} & =\boldsymbol{u}_{h}+\tilde{\boldsymbol{u}}, \\
p & =p_{h}+\tilde{p}, \\
\vartheta & =\vartheta_{h}+\tilde{\vartheta},
\end{aligned}
$$

where the components with subscript $h$ belong to the corresponding finite element spaces. The components with a tilde belong to any space such that its direct sum with the finite element space yields the functional space where the unknown is sought. For the moment, we leave it undefined. These additional components are what we will call subscales. Each particular variational multiscale method will depend on the way the subscales are approximated. Our main focus in this work is to explain the consequences of considering these subscales time dependent, and therefore requiring to be integrated in time. Likewise, we will keep the previous decomposition (12)-(14) in all the terms of the variational equations of the problem. As we shall see, this has important consequences in the modeling of thermally coupled turbulent flows. The only approximation we will make for the moment is to assume that the subscales vanish on the interelentent boundaries, $\partial \Omega_{e}$. This happens for example if one assumes that their Fourjer
modes correspond to high wave numbers, as it is explained in [6], but can be relaxed using the apprgach
proposed in [10].
From the previous splitting two sets of equations can be obtained. The first jo the projection of the
original equations onto the finite element spaces of velocity pres sure and temperature. On the other hand, the equations for the subscales are obtained by projecting onto their corresponding spaces, that is,

the projection onto any of the subscale spaces (for velocity, pressure or temperature), these equations are

$$
\begin{aligned}
\tilde{P}\left[\partial_{t} \tilde{\boldsymbol{u}}+\left(\boldsymbol{u}_{h}+\tilde{\boldsymbol{u}}\right) \cdot \nabla \tilde{\boldsymbol{u}}-\nu \Delta \tilde{\boldsymbol{u}}+\nabla \tilde{p}+\alpha \boldsymbol{g} \tilde{\vartheta}\right] & =\tilde{P}\left(\boldsymbol{R}_{u}\right), \\
\tilde{P}(\nabla \cdot \tilde{\boldsymbol{u}}) & =\tilde{P}\left(R_{p}\right), \\
\tilde{P}\left[\partial_{t} \tilde{\vartheta}+\left(\boldsymbol{u}_{h}+\tilde{\boldsymbol{u}}\right) \cdot \nabla \tilde{\vartheta}-\kappa \Delta \tilde{\vartheta}\right] & =\tilde{P}\left(R_{\vartheta}\right),
\end{aligned}
$$

where

$$
\begin{aligned}
\boldsymbol{R}_{u} & =\boldsymbol{f}+\alpha \boldsymbol{g} \vartheta_{0}-\left[\partial_{t} \boldsymbol{u}_{h}+\left(\boldsymbol{u}_{h}+\tilde{\boldsymbol{u}}\right) \cdot \nabla \boldsymbol{u}_{h}-\nu \Delta_{h} \boldsymbol{u}_{h}+\nabla p_{h}+\alpha \boldsymbol{g} \vartheta_{h}\right], \\
R_{p} & =-\nabla \cdot \boldsymbol{u}_{h}, \\
R_{\vartheta} & =Q-\left[\partial_{t} \vartheta_{h}+\left(\boldsymbol{u}_{h}+\tilde{\boldsymbol{u}}\right) \cdot \nabla \vartheta_{h}-\kappa \Delta_{h} \vartheta_{h}\right],
\end{aligned}
$$

are the residuals of the finite element unknowns in the momentum, continuity and heat equation, respectively. Equations (15)-(17) need to be solved within each element and, as we have assumed, considering homogeneous velocity and temperature Dirichlet boundary conditions.

\subsection{Approximation of the subscales I: general procedure}

In this subsection we present a general procedure to approximate the subscales in problem (15)-(17) and, in particular, of the spatial differential operators applied to the subgrid scales. To this end, let us 
consider an element $K$ of the finite element partition and a problem of the form

$$
\mathcal{L} u^{\prime}=r_{h} \quad \text { in } K,
$$

which needs to be completed with boundary conditions. As indicated previously, $u^{\prime}=0$ on $\partial K$ is a possibility. In the model problem (18), the unknown $u^{\prime}$ is assumed to have a vector character, although no particular notation will be used to specify it. Likewise, the forcing term $r_{h}$ is also a vector. The number of components of both $u^{\prime}$ and $r_{h}$ will be denoted by $n$.

Our objective is to obtain a $n \times n$ diagonal matrix $\tau$ such that

$$
u^{\prime} \approx \tau r_{h} \text { in each } K,
$$

so that $\tau \approx \mathcal{L}^{-1}$ with the appropriate boundary conditions. In the following subsection, this approximation will be used for the spatial operator arising from the linearization of the left-hand-side in (15)-(17), now $u^{\prime}$ being composed of the velocity components, the pressure and the temperature.

In order to obtain (19), we use a heuristic Fourier analysis, introduced in [6] and extended in [8], for

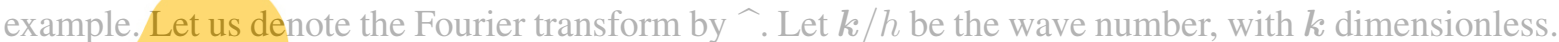
The basic heuristic assumption is that $u^{\prime}$ is highly fluctuating, and therefore dominated by high wave numbers. As a eonsequence, we may assume that

- Values of $u^{\prime}$ on $\partial K$ can be neglected to approximate $u^{\prime}$ in the interior of $K$.
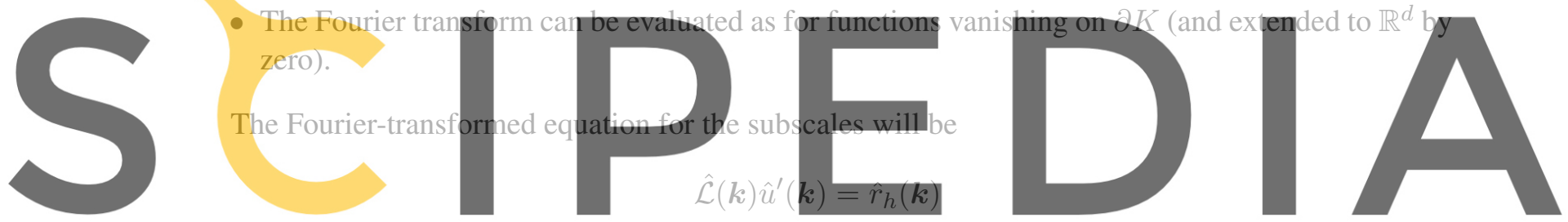

Before proceeding, it is crucial to discuss the proper scaling of this problem. Let $u$ be an element in

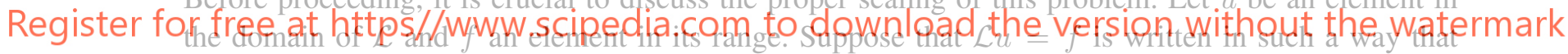

$f^{t} u=\sum_{i=1}^{n} f_{i} u_{i}$ is dimensionally well defined. In general, if $f, g \in$ range $\mathcal{L}$ and $u, v \in \operatorname{dom} \mathcal{L}$,

$$
f^{\mathrm{t}} g=\sum_{i=1}^{n} f_{i} g_{i}, \quad u^{\mathrm{t}} v=\sum_{i=1}^{n} u_{i} v_{i}
$$

may not be dimensionally meaningful. This is the case for example when the unknowns are $u=$ $(\boldsymbol{u}, p, \vartheta)$, as in our case.

Let $M$ be a scaling matrix, diagonal and with positive diagonal entries, that makes the products $f^{\mathrm{t}} M g$ and $u^{\mathrm{t}} M^{-1} v$ dimensionally consistent. Let also

$$
\begin{aligned}
|f|_{M}^{2} & :=f^{\mathrm{t}} M f \quad M \text {-norm of } f \\
|u|_{M^{-1}}^{2} & :=u^{\mathrm{t}} M^{-1} u \quad M^{-1} \text {-norm of } u \\
\|f\|_{L_{M}^{2}(K)} & :=\int_{K}|f|_{M}^{2}
\end{aligned}
$$

A simple stability analysis, which will be omitted here, dictates that matrix $\tau$ must be such that $\|\mathcal{L}\|_{L_{M}^{2}(K)} \leq\left\|\tau^{-1}\right\|_{L_{M}^{2}(K)}$. This will be the basic approximation condition of $\mathcal{L}$ by a diagonal ma- 
trix. In order to devise a way to satisfy it, let us note that

$$
\begin{aligned}
\|\mathcal{L} u\|_{L_{M}^{2}(K)}^{2} & =\int_{K}|\mathcal{L} u|_{M}^{2} \mathrm{~d} \boldsymbol{x} \\
& \approx \int_{\mathbb{R}^{d}}|\widehat{\mathcal{L}}(\boldsymbol{k}) \widehat{u}(\boldsymbol{k})|_{M}^{2} \mathrm{~d} \boldsymbol{k} \\
& \leq \int_{\mathbb{R}^{d}}|\widehat{\mathcal{L}}(\boldsymbol{k})|_{M}^{2}|\widehat{u}(\boldsymbol{k})|_{M}^{2} \mathrm{~d} \boldsymbol{k} \\
& =\left|\widehat{\mathcal{L}}\left(\boldsymbol{k}^{0}\right)\right|_{M}^{2} \int_{\mathbb{R}^{d}}|\widehat{u}(\boldsymbol{k})|_{M}^{2} \mathrm{~d} \boldsymbol{k} \\
& \approx\left|\widehat{\mathcal{L}}\left(\boldsymbol{k}^{0}\right)\right|_{M}^{2}\|u\|_{L_{M}^{2}(K)}^{2},
\end{aligned}
$$

where the first approximation comes from the fact that boundary values of $u^{\prime}$ have been discarded and $\boldsymbol{k}^{0}$ is a wave number whose existence follows from the mean value theorem. From the previous development we have that $\|\mathcal{L}\|_{L_{M}^{2}(K)} \leq\left|\widehat{\mathcal{L}}\left(k^{0}\right)\right|_{M}$. Our proposal is to choose $\tau$ diagonal and such that $\left|\widehat{\mathcal{L}}\left(\boldsymbol{k}^{0}\right)\right|_{M}=\left|\tau^{-1}\right|_{M}$. A particular way to achieve this is the following: let

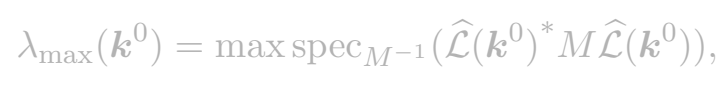

where $\lambda \in \operatorname{spec}_{M^{-1}} A$ if there exists $x$ such that $A x=\lambda M^{-1} x$. Then, we may require that
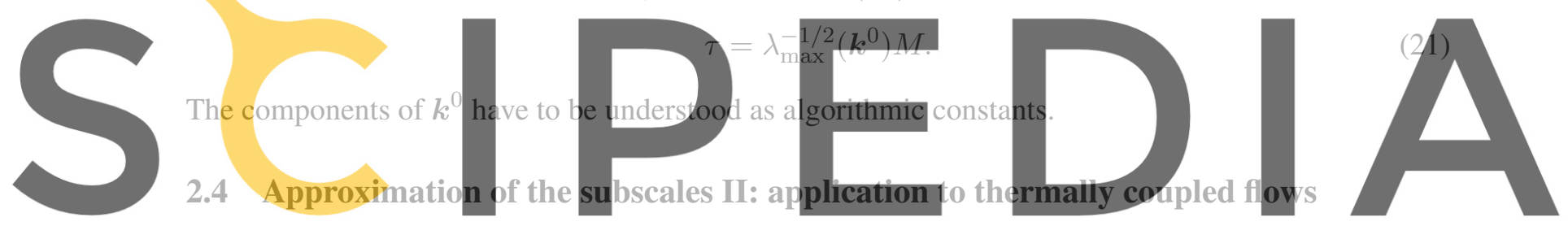

Let us apply the previous ideas to the spatial differential operator appearing in (15)-(17). If we call

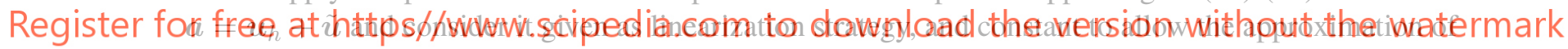
the Fourier transform, in the two-dimensional case it is found that

$$
\hat{\mathcal{L}}(\boldsymbol{k})=\left[\begin{array}{cccc}
\nu|\boldsymbol{k}|^{2}+\mathrm{i} a_{j} k_{j} & 0 & \mathrm{i} k_{1} & \alpha g_{1} s_{\vartheta} \\
0 & \nu|\boldsymbol{k}|^{2}+\mathrm{i} a_{j} k_{j} & \mathrm{i} k_{2} & \alpha g_{2} s_{\vartheta} \\
\mathrm{i} k_{1} & \mathrm{i} k_{2} & 0 & 0 \\
0 & 0 & 0 & s_{\vartheta}\left(\kappa|\boldsymbol{k}|^{2}+\mathrm{i} a_{j} k_{j}\right)
\end{array}\right],
$$

where $\mathrm{i}=\sqrt{-1}$ and $s_{\vartheta}$ is a scaling factor for the temperature such that $\boldsymbol{f} \cdot \boldsymbol{u}$ and $s_{\vartheta}^{-1} Q \vartheta$ have the same dimensions, that is to say, the dimensions of $s_{\vartheta}$ must be [Temperature] $]^{2}\left[\right.$ Velocity] ${ }^{-2}$. It could be for example $s_{\vartheta}=\delta \vartheta^{2} U^{-2}$, where $\delta \vartheta$ and $U$ are the characteristic values for temperature and velocity to define the dimensionless numbers in (11). The superscript in the wave number in (22) has been omitted.

Let us introduce the stabilization parameters $\tau_{1}, \tau_{2}$ and $\tau_{3}$, computed as

$$
\begin{aligned}
& \tau_{1}=\left[\left(c_{1} \frac{\nu}{h^{2}}\right)^{2}+\left(c_{2} \frac{\left|\boldsymbol{u}_{h}+\tilde{\boldsymbol{u}}\right|}{h}\right)^{2}\right]^{-1 / 2}, \\
& \tau_{2}=\frac{h^{2}}{c_{1} \tau_{1}}, \\
& \tau_{3}=\left[\left(c_{1} \frac{\kappa}{h^{2}}\right)^{2}+\left(c_{2} \frac{\left|\boldsymbol{u}_{h}+\tilde{\boldsymbol{u}}\right|}{h}\right)^{2}\right]^{-1 / 2},
\end{aligned}
$$


where $h$ is the element size and $c_{1}$ and $c_{2}$ are algorithmic constants (we have adopted $c_{1}=4$ and $c_{2}=2$ in the numerical experiments) that approximate $h^{2}|\boldsymbol{k}|^{2}$ and $h|\boldsymbol{k}|$ multiplied by the cosine of the angle formed by $\boldsymbol{k}$ with $\boldsymbol{u}_{h}+\tilde{\boldsymbol{u}}$.

A possible scaling matrix in this particular problem (in 2D) is

$$
M=\operatorname{diag}\left(\tau_{1}, \tau_{1}, \tau_{2}, \tau_{3} s_{\vartheta}^{-1}\right) .
$$

If this matrix is used to solve the eigenvalue problem in (20), with $\hat{\mathcal{L}}(\boldsymbol{k})$ given by (22), it is found that

$$
\lambda_{\max }=1+\frac{1}{2} \omega^{2}+\sqrt{\omega^{2}+\frac{1}{4} \omega^{4}}, \quad \omega^{2}:=\tau_{1} \tau_{3} \alpha^{2}|\boldsymbol{g}|^{2} s_{\vartheta} .
$$

Suppose that the problem has a characteristic velocity $U$. Let us identify with a subscript $h$ the analogous of the dimensionless numbers defined in (11) evaluated with the element size instead of $L$. If $s_{\vartheta}=\delta \vartheta^{2} U^{-2}$, as explained above, it may be readily seen that

\section{where $\sim$ stands for equality up to constants.}

\section{If the Boussinesq assumption is valid we may assume $\omega$ small, and expand}
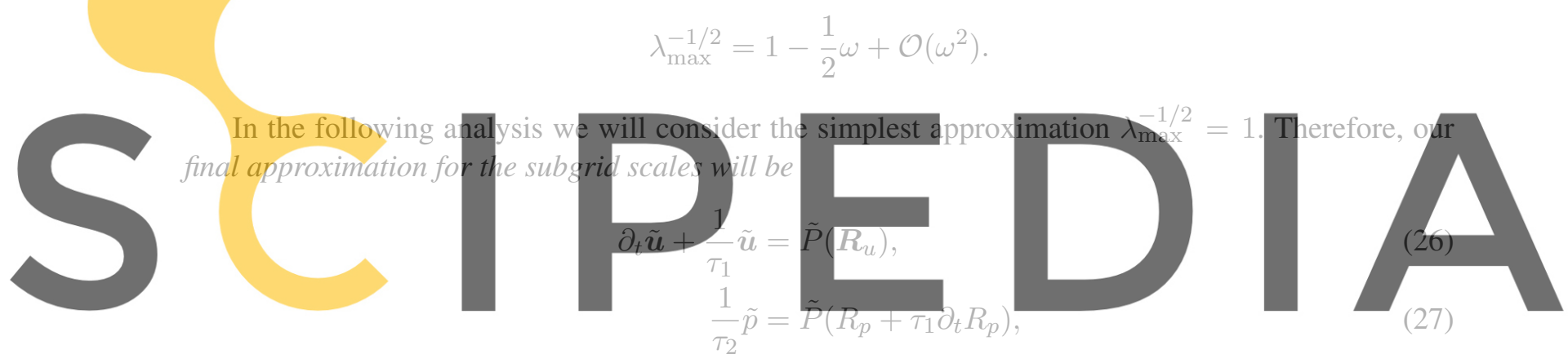

Register for free at https//www.scipegia.colmg to olqwyload the version without the wałermark

When the time derivative of the subscales is neglected, we will call them quasi-static, whereas otherwise we will call them dynamic.

\subsection{Final approximate problem in space}

Substituting (12)-(14) into (8)-(10), taking the test functions in the corresponding finite element spaces and integrating some terms by parts, and using the fact that $\boldsymbol{u}=\boldsymbol{u}_{h}+\tilde{\boldsymbol{u}}$ is divergence free, it is found that

$$
\begin{aligned}
& \left(\partial_{t} \boldsymbol{u}_{h}, \boldsymbol{v}_{h}\right)+\left\langle\boldsymbol{u}_{h} \cdot \nabla \boldsymbol{u}_{h}, \boldsymbol{v}_{h}\right\rangle+\nu\left(\nabla \boldsymbol{u}_{h}, \nabla \boldsymbol{v}_{h}\right)-\left(p_{h}, \nabla \cdot \boldsymbol{v}_{h}\right)+\alpha\left(\boldsymbol{g} \vartheta_{h}, \boldsymbol{v}_{h}\right) \\
& \quad-\left\langle\tilde{\boldsymbol{u}}, \nu \Delta_{h} \boldsymbol{v}_{h}+\boldsymbol{u}_{h} \cdot \nabla \boldsymbol{v}_{h}\right\rangle \\
& \quad+\left(\partial_{t} \tilde{\boldsymbol{u}}, \boldsymbol{v}_{h}\right)+\left\langle\tilde{\boldsymbol{u}} \cdot \nabla \boldsymbol{u}_{h}, \boldsymbol{v}_{h}\right\rangle-\left\langle\tilde{\boldsymbol{u}}, \tilde{\boldsymbol{u}} \cdot \nabla \boldsymbol{v}_{h}\right\rangle \\
& \quad-\left(\tilde{p}, \nabla \cdot \boldsymbol{v}_{h}\right)+\alpha\left(\boldsymbol{g} \tilde{\vartheta}, \boldsymbol{v}_{h}\right)=\left\langle\boldsymbol{f}, \boldsymbol{v}_{h}\right\rangle+\alpha\left(\boldsymbol{g} \vartheta_{0}, \boldsymbol{v}_{h}\right), \\
& \left(q_{h}, \nabla \cdot \boldsymbol{u}_{h}\right)-\left(\tilde{\boldsymbol{u}}, \nabla q_{h}\right)=0 \\
& \left(\partial_{t} \vartheta_{h}, \psi_{h}\right)+\left\langle\boldsymbol{u}_{h} \cdot \nabla \vartheta_{h}, \psi_{h}\right\rangle+\kappa\left(\nabla \vartheta_{h}, \nabla \psi_{h}\right) \\
& \quad-\left\langle\tilde{\vartheta}, \kappa \Delta_{h} \psi_{h}+\boldsymbol{u}_{h} \cdot \nabla \psi_{h}\right\rangle \\
& \quad+\left(\partial_{t} \tilde{\vartheta}, \psi_{h}\right)+\left\langle\tilde{\boldsymbol{u}} \cdot \nabla \vartheta_{h}, \psi_{h}\right\rangle-\left\langle\tilde{\vartheta}, \tilde{\boldsymbol{u}} \cdot \nabla \psi_{h}\right\rangle=\left\langle Q, \psi_{h}\right\rangle,
\end{aligned}
$$


which must hold for all test functions $\left(\boldsymbol{v}_{h}, q_{h}, \psi_{h}\right) \in \boldsymbol{V}_{h} \times Q_{h} \times \Psi_{h}$. The subindex $h$ in the Laplacian denotes that it is evaluated elementwise. The subscales in these equations are obtained from (26)-(28).

The first approximation involved in the previous equations is to assume that the subscales vanish at the interelement boundaries. The final numerical scheme is obtained by approximating these subscales in the element interiors, in our case by means of (26)-(28). These equations however still require the definition of the projections $\tilde{P}$. Classical stabilized finite element methods correspond to taking $\tilde{P}=I$ (identity) when applied to the corresponding finite element residual. Our proposal however is to take $\tilde{P}=P_{h}^{\perp}=I-P_{h}$, where $P_{h}$ is the $L^{2}$ projection onto the finite element space (see [6] and, for an analysis of the method for a stationary and linearized problem, [7]). This leads to what we call orthogonal subscale stabilization (OSS). When this is used in (29)-(31) one gets

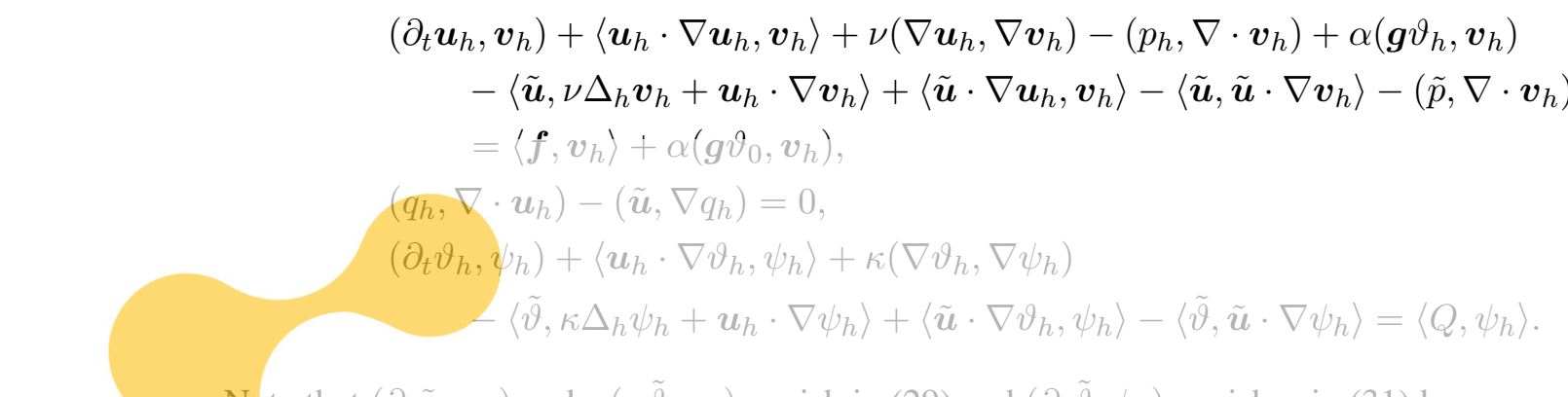

Note that $\left(\partial_{t} \tilde{\boldsymbol{u}}, \boldsymbol{v}_{h}\right)$ and $\alpha\left(\boldsymbol{g} \tilde{\vartheta}, \boldsymbol{v}_{h}\right)$ vanish in (29) and $\left(\partial_{t} \tilde{\vartheta}, \psi_{h}\right)$ vanishes in (31) because of the choice

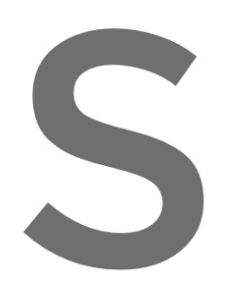
Any time discretization can now be applied to obtain a fully di
2.6 Main properties of the formulation
The first and most important point to be considered is the effect of
and therefore to deal with their time variation. Some of these prope
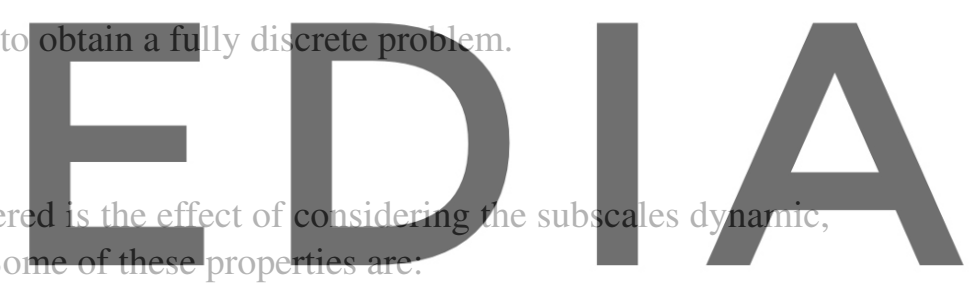

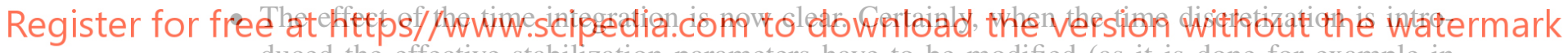
duced the effective stabilization parameters have to be modified (as it is done for example in $[2,26,27])$, but when the steady-state is reached the subscale $\tilde{u}$ that is obtained as solution to (26) satisfies $\tilde{\boldsymbol{u}}=\tau_{1} \tilde{P}\left(\boldsymbol{R}_{u}\right)$, so that the usual expression employed for stationary problems is recovered.

- Suppose for example that the backward Euler scheme is used to integrate (26). From the point of view of the algebraic solver, the factor $\left(\frac{1}{\delta t}+\frac{1}{\tau_{1}}\right)^{-1}$ instead of $\tau_{1}$ multiplying $\tilde{P}\left(\boldsymbol{R}_{u}\right)$ is crucial for the conditioning of the system matrix, since both for $\delta t \rightarrow 0$ and for $\delta t \rightarrow \infty$ the matrix contribution of the stabilization terms is dominated by the contribution from the Galerkin terms. If $\tau_{1}$ is used as stabilization factor, when $\delta t \rightarrow 0$ (and thus the leading terms are those coming from the discretization of the time derivative) both the Galerkin and stabilizing terms could lead to matrix terms of the same order and the condition number of the matrix of the Galerkin method could be deteriorated.

- It is clear that space discretization (understood as scale splitting) and time discretization commute, that is time discretization + stabilization (scale splitting) = stabilization (scale splitting) + time discretization.

- Numerical experiments show that the temporal time integration is significantly improved:

- Oscillations originated by initial transients are eliminated. 
- The numerical dissipation is minimized.

For the numerical results that demonstrate this fact we refer to $[11,9]$. This is also observed in the numerical experiments of Section 4.

- The numerical analysis shows optimal stability without any restriction between $\tau_{1}$ and $\delta t$. Contrary to classical stabilized methods, anisotropic space-time discretizations are allowed [3]. See [11] for a stability analysis of the linearized Navier-Stokes equations and [1] for a complete stability and convergence analysis for the Stokes problem.

Another very important issue of the formulation presented is the possibility to model turbulent flows. The terms involving the velocity subgrid scale arising from the convective term in the NavierStokes equations are $\left\langle\tilde{\boldsymbol{u}}, \tilde{\boldsymbol{u}} \cdot \nabla \boldsymbol{v}_{h}\right\rangle=\left\langle\nabla \boldsymbol{v}_{h}, \tilde{\boldsymbol{u}} \otimes \tilde{\boldsymbol{u}}\right\rangle$, which can be understood as the contribution from the Reynolds tensor an LES approach, and $-\left\langle\tilde{\boldsymbol{u}}, \boldsymbol{u}_{h} \cdot \nabla \boldsymbol{v}_{h}\right\rangle+\left\langle\tilde{\boldsymbol{u}} \cdot \nabla \boldsymbol{u}_{h}, \boldsymbol{v}_{h}\right\rangle$, which can be understood as the contribution from the cross stresses. Therefore, we may expect that, in some sense, modeling $\tilde{\boldsymbol{u}}$ implies to model the subgrid scale tensor. The question is how good this model will be. The numerical models proposed here yield two possibilities depending on the projection chosen, but others can be devised.

Related to the way turbulence is modeled, the numerical formulation proposed has an inherent turbulent Prandtl number. In other words, it is not necessary to specify which is the amount of turbulent thermal dissipation, but emanates directly from the formulation. This issue is further discussed later on.
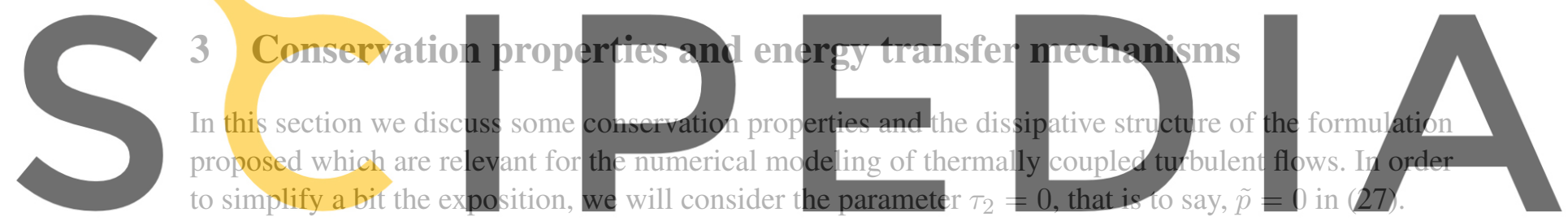

Another important remark is that the expression used for the convective term might not be the most

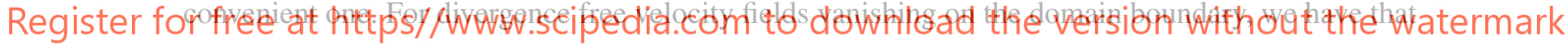

$$
\langle u \cdot \nabla u, v\rangle=-\langle u \otimes u, \nabla v\rangle=\frac{1}{2}\langle u \cdot \nabla u, v\rangle-\frac{1}{2}\langle u \otimes u, \nabla v\rangle .
$$

Any of these expressions can be used in the convective term of the approximate Navier-Stokes equations without altering the consistency. However, the discrete problem has different properties, as we will see. Thus, given a vector field $\boldsymbol{a}$ we introduce

$$
c_{u}(\boldsymbol{a} ; \boldsymbol{u}, \boldsymbol{v})= \begin{cases}c_{u}^{\mathrm{nc}}(\boldsymbol{a} ; \boldsymbol{u}, \boldsymbol{v})=\langle\boldsymbol{a} \cdot \nabla \boldsymbol{u}, \boldsymbol{v}\rangle & \text { Non conservative form } \\ c_{u}^{\mathrm{c}}(\boldsymbol{a} ; \boldsymbol{u}, \boldsymbol{v})=-\langle\boldsymbol{a} \otimes \boldsymbol{u}, \nabla \boldsymbol{v}\rangle & \text { Conservative form } \\ c_{u}^{\mathrm{ss}}(\boldsymbol{a} ; \boldsymbol{u}, \boldsymbol{v})=\frac{1}{2}\langle\boldsymbol{a} \cdot \nabla \boldsymbol{u}, \boldsymbol{v}\rangle-\frac{1}{2}\langle\boldsymbol{a} \otimes \boldsymbol{u}, \nabla \boldsymbol{v}\rangle & \text { Skew-symmetric form }\end{cases}
$$

Similarly, for the temperature equation we introduce

$$
c_{\vartheta}(\boldsymbol{a} ; \vartheta, \psi)= \begin{cases}c_{\vartheta}^{\mathrm{nc}}(\boldsymbol{a} ; \vartheta, \psi)=\langle\boldsymbol{a} \cdot \nabla \vartheta, \psi\rangle & \text { Non-conservative form } \\ c_{\vartheta}^{\mathrm{c}}(\boldsymbol{a} ; \vartheta, \psi)=-\langle\boldsymbol{a} \vartheta, \nabla \psi\rangle & \text { Conservative form } \\ c_{\vartheta}^{\mathrm{ss}}(\boldsymbol{a} ; \vartheta, \psi)=\frac{1}{2}\langle\boldsymbol{a} \cdot \nabla \vartheta, \psi\rangle-\frac{1}{2}\langle\boldsymbol{a} \vartheta, \nabla \psi\rangle & \text { Skew-symmetric form }\end{cases}
$$

The terms "conservative" and "non-conservative" are classical in the CFD community. The term "skewsymmetric" refers to the fact that

$$
c_{u}^{\mathrm{ss}}(\boldsymbol{a} ; \boldsymbol{u}, \boldsymbol{u})=0, \quad c_{\vartheta}^{\mathrm{sS}}(\boldsymbol{a} ; \vartheta, \vartheta)=0,
$$


even if $\boldsymbol{a}$ is not divergence free.

In order to study the conservation properties of the scheme, we consider the extended problem which includes the boundary fluxes $B_{R, u}\left(\boldsymbol{v}_{h}\right)$ and $B_{R, \vartheta}\left(\psi_{h}\right)$ in the Navier Stokes and heat equations [19]. These fluxes may include contributions from the convective term when $\boldsymbol{a}$ is not divergence free, which may change according to the form used for this term (non conservative, conservative or skew symmetric). This problem can be understood locally in a region $R$ formed by an arbitrary set of elements $[23,19]$, case in which boundary contributions come from the fluxes exchanged with the rest of the computational domain.

Using the approximation $\tau_{2}=0$, defining $\boldsymbol{a}=\boldsymbol{u}_{h}+\tilde{\boldsymbol{u}}$ (which is solenoidal prior to the approximation of the subscales), introducing the possibilities for the convective term described and accounting for the boundary fluxes, problem (32)-(34) can be reformulated as:

$$
\begin{aligned}
& \left(\partial_{t} \boldsymbol{u}_{h}, \boldsymbol{v}_{h}\right)+c_{u}\left(\boldsymbol{a} ; \boldsymbol{u}_{h}, \boldsymbol{v}_{h}\right)+\nu\left(\nabla \boldsymbol{u}_{h}, \nabla \boldsymbol{v}_{h}\right)-\left(p_{h}, \nabla \cdot \boldsymbol{v}_{h}\right)+\alpha\left(\boldsymbol{g} \vartheta_{h}, \boldsymbol{v}_{h}\right) \\
& \quad-\left\langle\tilde{u}, \nu \Delta_{h} \boldsymbol{v}_{h}+\boldsymbol{a} \cdot \nabla \boldsymbol{v}_{h}\right\rangle=\left\langle\boldsymbol{f}, \boldsymbol{v}_{h}\right\rangle+\alpha\left(\boldsymbol{g}_{0}, \boldsymbol{v}_{h}\right)+B_{R, u}\left(\boldsymbol{v}_{h}\right), \\
& \left(q_{h}, \nabla \cdot u_{h}\right)-\left(\tilde{u}, \nabla q_{h}\right)=0, \\
& \left(\partial_{t} \vartheta_{h}, \psi_{h}\right)+c_{\vartheta}\left(a ; \vartheta_{h}, \psi_{h}\right)+\kappa\left(\nabla \vartheta_{h}, \nabla \psi_{h}\right) \\
& \quad-\left\langle\vartheta_{,} \Delta_{h} \psi_{h}+a \cdot \nabla \psi_{h}\right\rangle=\left\langle Q, \psi_{h}\right\rangle+B_{R, \vartheta}\left(\psi_{h}\right) .
\end{aligned}
$$

As mentioned earlier, we may understand that this problem is posed in a region $R \subset \Omega$ formed by an arbitrary union of elements $K$ of the finite element partition. When $R=\Omega, B_{\Omega, u}\left(v_{h}\right)=0$ and
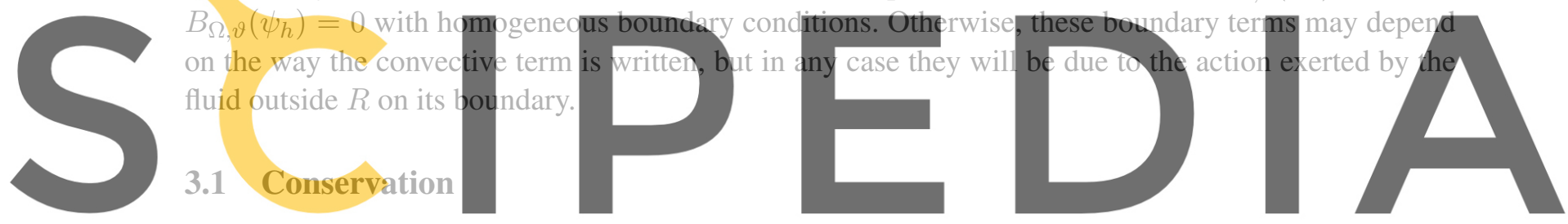

Different conservation statements can be obtained by taking appropriate test functions in the discrete

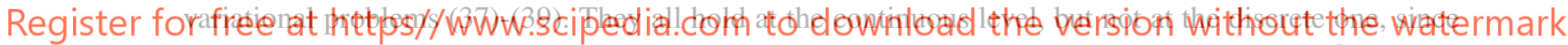
$a$ will not be exactly divergence free. In what follows, $R$ is considered strictly contained in $\Omega$ to allow us taking constant test functions in $R$. Otherwise, if $R=\Omega$ the homogeneous Dirichlet conditions have to be replaced by the appropriate fluxes.

\subsubsection{Conservation of linear momentum and heat}

Let $\boldsymbol{e}_{k}$ be the vector of $\mathbb{R}^{d}$ with the $k$-th component equal to 1 and the rest equal to 0 . Taking $\boldsymbol{v}_{h}=\boldsymbol{e}_{k}$ in (37) it follows that

$$
\frac{\mathrm{d}}{\mathrm{d} t} \int_{R} u_{h, k}+c_{u}\left(\boldsymbol{a} ; \boldsymbol{u}_{h}, \boldsymbol{e}_{k}\right)=\int_{R}\left[f_{k}+\alpha g_{k}\left(\vartheta_{0}-\vartheta_{h}\right)\right]+B_{R, u}\left(\boldsymbol{e}_{k}\right) .
$$

This equation can be understood as a conservation of linear momentum in a region $R$ provided $c_{u}\left(\boldsymbol{a} ; \boldsymbol{u}_{h}, \boldsymbol{e}_{k}\right)=0$ or has only contributions on $\partial R$. If the conservative form of the convective term is used it is obvious that $c_{u}^{\mathrm{c}}\left(\boldsymbol{a} ; \boldsymbol{u}_{h}, \boldsymbol{e}_{k}\right)=0$ (see (35)), so that the conservative form always conserves linear momentum. On the other hand, it is immediately checked that

$$
c_{u}^{\mathrm{nc}}\left(\boldsymbol{a} ; \boldsymbol{u}_{h}, \boldsymbol{e}_{k}\right)=-\int_{R} u_{h, k} \nabla \cdot \boldsymbol{u}_{h}+\int_{R} \tilde{\boldsymbol{u}} \cdot \nabla u_{h, k}+\int_{\partial R}\left(\boldsymbol{n} \cdot \boldsymbol{u}_{h}\right) u_{h, k},
$$


where $\boldsymbol{n}$ is the unit normal exterior to $\partial R$. From (38) it follows that the first two terms in this equation are zero, provided we can take $q_{h}=u_{h, k}$. Thus, the non-conservative form conserves linear momentum if equal velocity-pressure interpolations are used. Note that this would not be possible using the Galerkin method. This fact was already noticed in [19].

From the expression of the skew-symmetric form of the convective term it is clear that it has the same properties as the non-conservative form, since now

$$
c_{u}^{\mathrm{ss}}\left(\boldsymbol{a} ; \boldsymbol{u}_{h}, \boldsymbol{e}_{k}\right)=-\frac{1}{2} \int_{R} u_{h, k} \nabla \cdot \boldsymbol{u}_{h}+\frac{1}{2} \int_{R} \tilde{\boldsymbol{u}} \cdot \nabla u_{h, k}+\frac{1}{2} \int_{\partial R}\left(\boldsymbol{n} \cdot \boldsymbol{u}_{h}\right) u_{h, k} .
$$

A similar analysis can be undertaken for the heat equation. If $\psi_{h}=1$ in (39) it follows that

$$
\frac{\mathrm{d}}{\mathrm{d} t} \int_{R} \vartheta_{h}+c_{\vartheta}\left(\boldsymbol{a} ; \vartheta_{h}, 1\right)=\int_{R} Q+B_{R, \vartheta}(1) .
$$

This equation can be understood as a conservation of heat in a region $R$ provided $c_{\vartheta}\left(\boldsymbol{a} ; \vartheta_{h}, 1\right)=0$ or contributes only with terms defined on $\partial R$. Once again, if the conservative form of the convective term is used, $c_{\vartheta}^{\mathrm{c}}\left(\boldsymbol{a} ; \vartheta_{h}, 1\right)=0$ (see (36)), so that the conservative form always conserves heat. On the other hand,

$$
c_{\vartheta}^{\mathrm{nc}}\left(\boldsymbol{a} ; \vartheta_{h}, 1\right)=-\int_{R} \vartheta_{h} \nabla \cdot \boldsymbol{u}_{h}+\int_{R} \tilde{\boldsymbol{u}} \cdot \nabla \vartheta_{h}+\int_{\partial R}\left(\boldsymbol{n} \cdot \boldsymbol{u}_{h}\right) \vartheta_{h} .
$$

From (38) it follows that the first two terms in this equation are zero, provided we can take $q_{h}=\vartheta_{h}$. Thus, the non-conservative form conserves heat if equal temperature-pressure interpolations are used. As for the Navier-Stokes equations, the same conclusion applies to the skew-symmetric form of the convective term in the heat equation.

\subsubsection{Conservation of angular momentum}

Conservation of angular momentum for the continuous version of (37) is obtained by taking $\boldsymbol{v}=\boldsymbol{e}_{k} \times \boldsymbol{x}$, where $\boldsymbol{x}$ is the position vector. For all vectors $\boldsymbol{w}$ it follows that $\boldsymbol{w} \cdot \boldsymbol{v}=\boldsymbol{x} \times\left.\boldsymbol{w}\right|_{k}$ (the $k$-th component of $\boldsymbol{x} \times \boldsymbol{w})$. Note also that $\nabla \cdot\left(\boldsymbol{e}_{k} \times \boldsymbol{x}\right)=0$.

If we take $\boldsymbol{v}_{h}=\boldsymbol{e}_{k} \times \boldsymbol{x}$, a necessary condition to have a global angular momentum conservation statement is that the viscous term vanishes. It is readily checked that

$$
\nu\left(\nabla \boldsymbol{u}_{h}, \nabla\left(\boldsymbol{e}_{k} \times \boldsymbol{x}\right)\right)=\nu \int_{R} \nabla \times\left.\boldsymbol{u}_{h}\right|_{k},
$$

which is in general not zero. Let $\nabla^{S}$ be the symmetrical gradient operator. The viscous term at the continuous level could also be written as $2 \nu\left(\nabla^{S} \boldsymbol{u}, \nabla^{S} \boldsymbol{v}\right)$. Since $\nabla^{S}\left(\boldsymbol{e}_{k} \times \boldsymbol{x}\right)=\mathbf{0}$, we conclude that the viscous term has to be written as $2 \nu\left(\nabla^{S} \boldsymbol{u}_{h}, \nabla^{S} \boldsymbol{v}_{h}\right)$ to allow global conservation of angular momentum. However, writing the viscous term this way is obviously not enough. The convective term must also vanish when $\boldsymbol{v}_{h}=\boldsymbol{e}_{k} \times \boldsymbol{x}$ (or lead only to boundary contributions). If the non-conservative form is used we have that

$$
c_{u}^{\mathrm{nc}}\left(\boldsymbol{a} ; \boldsymbol{u}_{h}, \boldsymbol{e}_{k} \times \boldsymbol{x}\right)=-\int_{R}\left(\nabla \cdot \boldsymbol{u}_{h}\right) \boldsymbol{x} \times\left.\boldsymbol{u}_{h}\right|_{k}+\int_{R} \tilde{\boldsymbol{u}} \cdot \nabla\left(\boldsymbol{x} \times\left.\boldsymbol{u}_{h}\right|_{k}\right)+\int_{\partial R}\left(\boldsymbol{n} \cdot \boldsymbol{u}_{h}\right) \boldsymbol{x} \times\left.\boldsymbol{u}_{h}\right|_{k} .
$$

We could guarantee that the first two terms vanish only if we could take $q_{h}=\boldsymbol{x} \times\left.\boldsymbol{u}_{h}\right|_{k}$, which would be possible only if the pressure interpolation is of one order higher than the velocity interpolation. This does not make sense for the approximation of the Navier-Stokes equations, and therefore we consider 
not possible to have angular momentum conservation using the non-conservative form of the convective term. However, we can take $q_{h}=P_{h}\left(\boldsymbol{x} \times\left.\boldsymbol{u}_{h}\right|_{k}\right)$ in (38), and therefore (40) reduces to

$$
\begin{aligned}
c_{u}^{\mathrm{nc}}\left(\boldsymbol{a} ; \boldsymbol{u}_{h}, \boldsymbol{e}_{k} \times \boldsymbol{x}\right)= & -\int_{R}\left(\nabla \cdot \boldsymbol{u}_{h}\right) P_{h}^{\perp}\left(\boldsymbol{x} \times\left.\boldsymbol{u}_{h}\right|_{k}\right)+\int_{R} \tilde{\boldsymbol{u}} \cdot \nabla P_{h}^{\perp}\left(\boldsymbol{x} \times\left.\boldsymbol{u}_{h}\right|_{k}\right) \\
& +\int_{\partial R}\left(\boldsymbol{n} \cdot \boldsymbol{u}_{h}\right) \boldsymbol{x} \times\left.\boldsymbol{u}_{h}\right|_{k},
\end{aligned}
$$

and, formally, $P_{h}^{\perp}\left(\boldsymbol{x} \times\left.\boldsymbol{u}_{h}\right|_{k}\right)$ is of order $r+1, r$ being the interpolation order. Thus, the error involved in the approximation of the angular momentum will be small. Concerning the contribution from the velocity subscales in (37), it holds

$$
-\left\langle\tilde{\boldsymbol{u}}, \boldsymbol{a} \cdot \nabla\left(\boldsymbol{e}_{k} \times \boldsymbol{x}\right)\right\rangle=-\int_{R} \tilde{\boldsymbol{u}} \times\left.\boldsymbol{u}_{h}\right|_{k}
$$

which is zero if the subscales are $L^{2}$ orthogonal to the finite element space. Clearly, the same comments apply to the skew-symmetric form of the convective term.

Let us move our attention to the conservative form defined in (35). It is readily checked that

$$
c_{u}^{\mathrm{c}}\left(\boldsymbol{a} ; \boldsymbol{u}_{h}, \boldsymbol{e}_{k} \times \boldsymbol{x}\right)=\int_{R} \tilde{\boldsymbol{u}} \times\left.\boldsymbol{u}_{h}\right|_{k},
$$

This, together with (41), yields

$$
c_{u}^{\mathrm{c}}\left(\boldsymbol{a} ; \boldsymbol{u}_{h}, \boldsymbol{e}_{k} \times \boldsymbol{x}\right)-\left\langle\tilde{\boldsymbol{u}}, \boldsymbol{a} \cdot \nabla\left(\boldsymbol{e}_{k} \times \boldsymbol{x}\right)\right\rangle=0,
$$

so that we obtain the statement of angular momentum conservation

$$
\frac{\mathrm{d}}{\mathrm{d} t} \int_{R} \boldsymbol{x} \times \boldsymbol{u}_{h}=\int_{R} \boldsymbol{x} \times\left[\boldsymbol{f}+\alpha \boldsymbol{g}\left(\vartheta_{0}-\vartheta_{h}\right)\right]+B_{R, u}\left(\boldsymbol{e}_{k} \times \boldsymbol{x}\right) .
$$

when the conservation form of the convective term is employed, independently of whether the velocity subscales are orthogonal or not to the finite element space.

\subsubsection{Conservation of kinetic energy and heat energy}

The last conservation statements we wish to discuss are those of kinetic energy for the Navier-Stokes equations and of heat energy for the heat equation. By "energy" we mean simply the $L^{2}$ norm of the velocity or the temperature, although in order to obtain quantities with dimension of energy these norms have to be properly scaled. For the continuous problem, conservation of these quantities is obtained by taking the test functions equal to the velocity and the temperature, respectively, and using in a crucial manner the fact that the velocity is solenoidal to conclude that the convective terms in the corresponding equations do not contribute.

In the discrete case, we need to have $c_{u}\left(\boldsymbol{a} ; \boldsymbol{u}_{h}, \boldsymbol{u}_{h}\right)=0$ and $c_{\vartheta}\left(\boldsymbol{a} ; \vartheta_{h}, \vartheta_{h}\right)=0$. This is automatically satisfied for the skew-symmetric forms of these convective terms (this leads in fact to their definition), but not for the conservative or non-conservative forms. Therefore, only the skew-symmetric expressions in (35) and (36) may lead to conservation of kinetic energy and of heat energy, respectively.

When obtaining energy balance statements is when the importance of orthogonal and dynamic subgrid scales is more evident. To this end, it is enlightening not only to take $\boldsymbol{v}_{h}=\boldsymbol{u}_{h}, q_{h}=p_{h}$ and 
$\psi_{h}=\vartheta_{h}$ (for each $t \in(0, T)$ ), but also to test the equations for the subscales (26) and (28) (recall that we are assuming $\tau_{2}=0$ ) by $\tilde{\boldsymbol{u}}$ and $\tilde{\vartheta}$, respectively. If this is done, we get:

$$
\begin{array}{r}
\frac{1}{2} \frac{\mathrm{d}}{\mathrm{d} t}\left\|\boldsymbol{u}_{h}\right\|_{R}^{2}+\nu\left\|\nabla \boldsymbol{u}_{h}\right\|_{R}^{2}-\sum_{K \subset R}\left\langle\tilde{\boldsymbol{u}}, \tilde{P}\left(\nu \Delta_{h} \boldsymbol{u}_{h}+\boldsymbol{a} \cdot \nabla \boldsymbol{u}_{h}+\nabla p_{h}\right)\right\rangle_{K}=W_{h}, \\
\frac{1}{2} \frac{\mathrm{d}}{\mathrm{d} t}\|\tilde{\boldsymbol{u}}\|_{R}^{2}+\tau_{1}^{-1}\|\tilde{\boldsymbol{u}}\|_{R}^{2}-\sum_{K \subset R}\left\langle\tilde{\boldsymbol{u}}, \tilde{P}\left(\nu \Delta_{h} \boldsymbol{u}_{h}-\boldsymbol{a} \cdot \nabla \boldsymbol{u}_{h}-\nabla p_{h}\right)\right\rangle_{K}=\tilde{W}, \\
\frac{1}{2} \frac{\mathrm{d}}{\mathrm{d} t}\left\|\vartheta_{h}\right\|_{R}^{2}+\kappa\left\|\nabla \vartheta_{h}\right\|_{R}^{2}-\sum_{K \subset R}\left\langle\tilde{\vartheta}, \tilde{P}\left(\kappa \Delta_{h} \vartheta_{h}+\boldsymbol{a} \cdot \nabla \vartheta_{h}\right)\right\rangle_{K}=H_{h}, \\
\frac{1}{2} \frac{\mathrm{d}}{\mathrm{d} t}\|\tilde{\vartheta}\|_{R}^{2}+\tau_{3}^{-1}\|\tilde{\vartheta}\|_{R}^{2}-\sum_{K \subset R}\left\langle\tilde{\vartheta}, \tilde{P}\left(\kappa \Delta_{h} \vartheta_{h}-\boldsymbol{a} \cdot \nabla \vartheta_{h}\right)\right\rangle_{K}=\tilde{H} .
\end{array}
$$

In these expressions, $\|\cdot\|_{R}$ is the $L^{2}$ norm in $R,\langle f, g\rangle_{K}=\int_{K} f g, W_{h}$ is the total mechanical power on $R$ due to $\boldsymbol{u}_{h}$ (including the contribution from the Boussinesq model), $\tilde{W}$ the total mechanical power on $R$ due to $\tilde{\boldsymbol{u}}, H_{h}$ the total heat power on $R$ due to $\vartheta_{h}$ and $\tilde{H}$ the total heat power on $R$ due to $\tilde{\vartheta}$. Here and in what follows we have assumed the stabilization parameters $\tau_{1}$ and $\tau_{3}$ constant in region $R$.

It is obvious from (43)-(46) that there is no balance statement for the kinetic energy of $\boldsymbol{u}_{h}$ or the heat energy of $\vartheta_{h}$ alone, in the form of time variation plus dissipation equal to external input. However, these balance statements can indeed be found when the contributions from the finite element components and the subscales are added up. We will further elaborate this point in the following subsection, but we may already notice that

$$
\begin{gathered}
\frac{1}{2} \frac{\mathrm{d}}{\mathrm{d} t}\left\|\boldsymbol{u}_{h}\right\|_{R}^{2}+\frac{1}{2} \frac{\mathrm{d}}{\mathrm{d} t}\|\tilde{\boldsymbol{u}}\|_{R}^{2}+\nu\left\|\nabla \boldsymbol{u}_{h}\right\|_{R}^{2}+\tau_{1}^{-1}\|\tilde{\boldsymbol{u}}\|_{R}^{2}-2 \sum_{K \subset R}\left\langle\tilde{\boldsymbol{u}}, \tilde{P}\left(\nu \Delta_{h} \boldsymbol{u}_{h}\right)\right\rangle_{K}=W_{h}+\tilde{W}, \\
\frac{1}{2} \frac{\mathrm{d}}{\mathrm{d} t}\left\|\vartheta_{h}\right\|_{R}^{2}+\frac{1}{2} \frac{\mathrm{d}}{\mathrm{d} t}\|\tilde{\vartheta}\|_{R}^{2}+\kappa\left\|\nabla \vartheta_{h}\right\|_{R}^{2}+\tau_{3}^{-1}\|\tilde{\vartheta}\|_{R}^{2}-2 \sum_{K \subset R}\left\langle\tilde{\vartheta}, \tilde{P}\left(\kappa \Delta_{h} \vartheta_{h}\right)\right\rangle_{K}=H_{h}+\tilde{H}
\end{gathered}
$$

The second order derivatives can be neglected for linear interpolations (they identically zero) or when $\tilde{P}$ is taken as the $L^{2}$ projection to the space orthogonal to the corresponding finite element space (of velocities or of temperatures) without boundary conditions. In any case, from the expression of the stabilization parameters and some simple inverse estimates it can be shown that

$$
\nu\left\|\nabla \boldsymbol{u}_{h}\right\|_{R}^{2}+\tau_{1}^{-1}\|\tilde{\boldsymbol{u}}\|_{R}^{2}-2 \sum_{K \subset R}\left\langle\tilde{\boldsymbol{u}}, \tilde{P}\left(\nu \Delta_{h} \boldsymbol{u}_{h}\right)\right\rangle_{K} \geq C\left(\nu\left\|\nabla \boldsymbol{u}_{h}\right\|_{R}^{2}+\tau_{1}^{-1}\|\tilde{\boldsymbol{u}}\|_{R}^{2}\right),
$$

for a constant $C>0$, and similarly for the heat equation. Therefore, (47)-(48) do have the structure of time variation of energy plus dissipation equal to external input. In equations (43)-(46) there are some additional terms that can be understood as transfer of energy between scales, as explained in Subsection 3.2 below.

\subsubsection{Summary}

The results obtained in this subsection are collected in Table 1 for the Navier-Stokes equations and in Table 2 for the heat equation. As we have seen, the crucial point to obtain these results is the way in which the convective term is written.

Concerning the conservation of energy, it has to be understood that this refers to a balance in the sense described above, that is, including a positive dissipative term. As we have seen, this applies to the sum of the finite element component and the subscales (of velocity and of temperature), whereas the 


\begin{tabular}{|c||c|c|c|}
\hline Convective term & Linear momentum & Angular momentum & Kinetic energy \\
\hline \hline Conservative & Yes & Yes & No \\
\hline Non-conservative & $\begin{array}{c}\text { Yes } \\
\text { With equal } \boldsymbol{u} \text { - } \boldsymbol{p} \text { interpolation }\end{array}$ & No & No \\
\hline Skew-symmetric & $\begin{array}{c}\text { Yes } \\
\text { With equal } \boldsymbol{u}-\boldsymbol{p} \text { interpolation }\end{array}$ & No & Yes \\
\hline
\end{tabular}

Table 1: Conservation properties for the Navier-Stokes equations depending on the expression of the convective term

\begin{tabular}{|c||c|c|}
\hline Convective term & Heat & Heat energy \\
\hline \hline Conservative & Yes & No \\
\hline Non-conservative & $\begin{array}{c}\text { Yes } \\
\text { With equal } p \text { - } \text { interpolation }\end{array}$ & No \\
\hline Skew-symmetric & $\begin{array}{c}\text { Yes } \\
\text { With equal } p \text { - } \vartheta \text { interpolation }\end{array}$ & Yes \\
\hline
\end{tabular}

Table 2: Conservation properties for the heat equation depending on the expression of the convective term

rest of conservation statements apply to the finite element component only. In fact, from the expression of the approximate equations for the subscales, (26) and (28), it can be seen that neither linear momentum nor angular momentum can be conserved for $\tilde{\boldsymbol{u}}$ (both will always decrease) and heat cannot be conserved for $\tilde{\vartheta}$ (it will also decrease).

\subsection{Energy transfer terms}

Let us take a closer look at the energy conservation equations (43)-(46). Introducing the definitions of Table 3, these equations can be written as

$$
\begin{aligned}
& \frac{\mathrm{d}}{\mathrm{d} t} \mathcal{E}_{h}^{u}+\mathcal{D}_{h}^{u}+\mathcal{C}^{u}+\mathcal{T}^{u}=W_{h}, \\
& \frac{\mathrm{d}}{\mathrm{d} t} \tilde{\mathcal{E}}^{u}+\tilde{\mathcal{D}}^{u}+\mathcal{C}^{u}-\mathcal{T}^{u}=\tilde{W}, \\
& \frac{\mathrm{d}}{\mathrm{d} t} \mathcal{E}_{h}^{\vartheta}+\mathcal{D}_{h}^{\vartheta}+\mathcal{C}^{\vartheta}+\mathcal{T}^{\vartheta}=H_{h}, \\
& \frac{\mathrm{d}}{\mathrm{d} t} \tilde{\mathcal{E}}^{\vartheta}+\tilde{\mathcal{D}}^{\vartheta}+\mathcal{C}^{\vartheta}-\mathcal{T}^{\vartheta}=\tilde{H} .
\end{aligned}
$$

Note that with the Boussinesq model there is an energy input in the Navier-Stokes equations in the form of external power, in our case contained in the term $W_{h}$, that is not reflected in the heat equation.

Several remarks are in order:

- The dissipation terms are strictly positive. They contribute to decrease the energy of the variable whose balance is expressed in the equation where they appear.

- The cross scale dissipation terms defined in Table 3 appear in both the equation for the finite element scale and for the subscale (in either the Navier-Stokes or the heat equation). As explained earlier, they can be absorbed by the dissipation of both the finite element scale and the subscale, 


\begin{tabular}{|c||c|c|}
\hline Equation & Energy & Dissipation \\
\hline \hline $\boldsymbol{u}_{h}$ & $\mathcal{E}_{h}^{u}=\frac{1}{2}\left\|\boldsymbol{u}_{h}\right\|_{R}^{2}$ & $\mathcal{D}_{h}^{u}=\nu\left\|\nabla \boldsymbol{u}_{h}\right\|_{R}^{2}$ \\
\hline$\tilde{\boldsymbol{u}}$ & $\tilde{\mathcal{E}}^{u}=\frac{1}{2}\|\tilde{\boldsymbol{u}}\|_{R}^{2}$ & $\tilde{\mathcal{D}}^{u}=\tau_{1}^{-1}\|\tilde{\boldsymbol{u}}\|_{R}^{2}$ \\
\hline$\vartheta_{h}$ & $\mathcal{E}_{h}^{\vartheta}=\frac{1}{2}\left\|\vartheta_{h}\right\|_{R}^{2}$ & $\mathcal{D}_{h}^{\vartheta}=\kappa\left\|\nabla \vartheta_{h}\right\|_{R}^{2}$ \\
\hline$\tilde{\vartheta}$ & $\tilde{\mathcal{E}}^{\vartheta}=\frac{1}{2}\|\tilde{\vartheta}\|_{R}^{2}$ & $\tilde{\mathcal{D}}^{\vartheta}=\tau_{3}^{-1}\|\tilde{\vartheta}\|_{R}^{2}$ \\
\hline \hline Equation & Cross scale dissipation & Transfer term \\
\hline \hline $\boldsymbol{u}_{h}$ & $\mathcal{C}^{u}=-\sum_{K \subset R}\left\langle\tilde{\boldsymbol{u}}, \tilde{P}\left(\nu \Delta_{h} \boldsymbol{u}_{h}\right)\right\rangle_{K}$ & $\mathcal{T}^{u}=-\sum_{K \subset R}\left\langle\tilde{\boldsymbol{u}}, \tilde{P}\left(\boldsymbol{a} \cdot \nabla \boldsymbol{u}_{h}+\nabla p_{h}\right)\right\rangle_{K}$ \\
\hline$\tilde{\boldsymbol{u}}$ & $\mathcal{C}^{u}$ & $-\mathcal{T}^{u}$ \\
\hline$\vartheta_{h}$ & $\mathcal{C}^{\vartheta}=-\sum_{K \subset R}\left\langle\tilde{\vartheta}, \tilde{P}\left(\kappa \Delta_{h} \vartheta_{h}\right)\right\rangle_{K}$ & $\mathcal{T}^{\vartheta}=-\sum_{K \subset R}\left\langle\tilde{\vartheta}, \tilde{P}\left(\boldsymbol{a} \cdot \nabla \vartheta_{h}\right)\right\rangle_{K}$ \\
\hline$\tilde{\vartheta}$ & $\mathcal{C}^{\vartheta}$ & $-\mathcal{T}^{\vartheta}$ \\
\hline
\end{tabular}

Table 3: Energy transfer terms

but not by any of them independently. Therefore, the cross scale dissipation terms couple the energy balance of the two scales of the problem. However, these terms vanish as $\nu \rightarrow 0$ and $\kappa \rightarrow 0$, and are otherwise active when viscosity and conductivity are high. In this case it is known that there is no scale separation, because the flow is completely resolved, i.e. direct numerical simulation (DNS) resolution has been reached.

- The transfer terms appear with an opposite sign in the energy equation for the finite element component and the subscale component. Thus, they certainly represent transfer of energy between scales. This, together with the fact that the cross scale dissipation terms vanish for vanishing viscosity and conductivity, leads us to conclude that in this situation there is a scale separation between the finite element components and the subscales. To arrive to this conclusion, it is essential to consider the subscales dynamic and orthogonal to the finite element space.

\subsection{Numerical dissipation}

To conclude this section, let us discuss the concept of numerical dissipation of the algorithm, both for the Navier-Stokes and the heat equation, and the possibility to model turbulence using this dissipation.

One can consider as numerical dissipation the one that affects the finite element component alone. If we write the subscales emanating from (26) and (28) as

$$
\begin{aligned}
& \tilde{\boldsymbol{u}}=\tau_{1}\left(\tilde{P}\left(\boldsymbol{R}_{u}\right)-\partial_{t} \tilde{\boldsymbol{u}}\right), \\
& \tilde{\vartheta}=\tau_{3}\left(\tilde{P}\left(R_{\vartheta}\right)-\partial_{t} \tilde{\vartheta}\right),
\end{aligned}
$$

we may write the total dissipation of the finite element scales as

$$
\begin{aligned}
& \varepsilon_{\text {num }}^{u}:=-\tau_{1} \sum_{K \subset R}\left\langle\tilde{P}\left(\boldsymbol{R}_{u}\right)-\partial_{t} \tilde{\boldsymbol{u}}, \tilde{P}\left(\nu \Delta_{h} \boldsymbol{u}_{h}+\boldsymbol{a} \cdot \nabla \boldsymbol{u}_{h}+\nabla p_{h}\right)\right\rangle_{K}, \\
& \varepsilon_{\mathrm{num}}^{\vartheta}:=-\tau_{3} \sum_{K \subset R}\left\langle\tilde{P}\left(R_{\vartheta}\right)-\partial_{t} \tilde{\vartheta}, \tilde{P}\left(\kappa \Delta_{h} \vartheta_{h}-\boldsymbol{a} \cdot \nabla \vartheta_{h}\right)\right\rangle_{K} .
\end{aligned}
$$


There are two main properties of $\varepsilon_{\text {num }}^{u}$ that are of paramount importance in the modeling of turbulent flows:

- For quasi-static subscales, it is shown in [13] that $\varepsilon_{\text {num }}^{u}$ behaves as the molecular dissipation of the continuous problem when the assumptions of classical statistical fluid mechanics apply and the mesh size $h$ belongs to the inertial range of the Kolmogorov spectrum. This is precisely the requirement posed by Lilly to LES models [20], and thus it poses the question of whether additional LES modeling is required or not in our pure numerical approach. Our claim is that the answer is no.

- For quasi-static subscales, $\boldsymbol{f}=\mathbf{0}$ (or it is a finite element function) and $\nu \rightarrow 0$ and, $\varepsilon_{\text {num }}^{u}$ is non-negative at each point and at each time instant. However, for dynamic subscales this cannot be guaranteed a priori. In fact, numerical experiments show that $\varepsilon_{\text {num }}^{u}$ can be negative at some points and some time instants [23], that is to say, dynamic subscales allow for backscatter.

When applicable, similar concepts can be applied to the dissipation of the heat equation, $\varepsilon_{\text {num }}^{\vartheta}$. However, in this case there is an additional issue to consider, namely, which is the ratio between $\varepsilon_{\text {num }}^{u}$ and $\varepsilon_{\text {num }}^{\vartheta}$, after appropriate scaling. This is what can be considered the turbulent Prandtl number. In usual LES models it needs to be assumed a priori. In our case it is an outcome of the numerical model.

If we introduce the effective turbulent viscosity and turbulent thermal conductivity

$$
\nu_{\text {tur }}=\frac{\varepsilon_{\text {num }}^{u}}{\left\|\nabla \boldsymbol{u}_{h}\right\|^{2}}, \quad \kappa_{\text {tur }}=\frac{\varepsilon_{\text {num }}^{\vartheta}}{\left\|\nabla \vartheta_{h}\right\|^{2}},
$$

the turbulent Prandtl number may be defined as

$$
\operatorname{Pr}_{\text {tur }}:=\frac{\nu_{\text {tur }}}{\kappa_{\text {tur }}}=\frac{\varepsilon_{\text {num }}^{u}}{\left\|\nabla \boldsymbol{u}_{h}\right\|^{2}} \frac{\left\|\nabla \vartheta_{h}\right\|^{2}}{\varepsilon_{\text {num }}^{\vartheta}} .
$$

In view of expressions (49) and (50), if for $\nu \rightarrow 0$ and $\kappa \rightarrow 0$ we neglect the influence of the pressure gradient and assume that the gradients of velocity and temperature form the same angle with $\boldsymbol{a}$, we may estimate

$$
\operatorname{Pr}_{\text {tur }}^{2} \sim \frac{\tau_{1}^{2}}{\tau_{3}^{2}}=\frac{c_{1}^{2} \kappa^{2}+c_{2}^{2}|\boldsymbol{a}|^{2} h^{2}}{c_{1}^{2} \nu^{2}+c_{2}^{2}|\boldsymbol{a}|^{2} h^{2}}=\frac{1+\frac{c_{2}^{2}}{c_{1}^{2}} \mathrm{Pe}_{h}^{2}}{\operatorname{Pr}^{2}+\frac{c_{2}^{2}}{c_{1}^{2}} \mathrm{Pe}_{h}^{2}},
$$

where

$$
\mathrm{Pe}_{h}:=\frac{|\boldsymbol{a}| h}{\kappa},
$$

is the element Péclet number. From (52) it follows that

$$
\operatorname{Pr}_{\text {tur }} \sim \begin{cases}\frac{1}{\operatorname{Pr}} & \text { if } \mathrm{Pe}_{h} \rightarrow 0 \\ 1 & \text { if } \mathrm{Pe}_{h} \rightarrow \infty\end{cases}
$$

These limiting situations cannot be assumed in general turbulent flows. In a numerical example we will show that in fact the effective turbulent Prandtl number departs significantly from $\operatorname{Pr}_{\text {tur }}=1$, which is the value usually adopted when modeling turbulent thermal flows. 

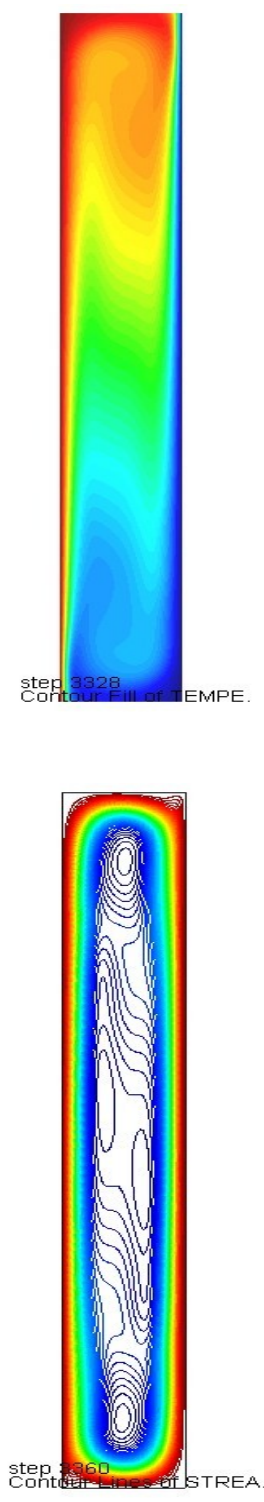
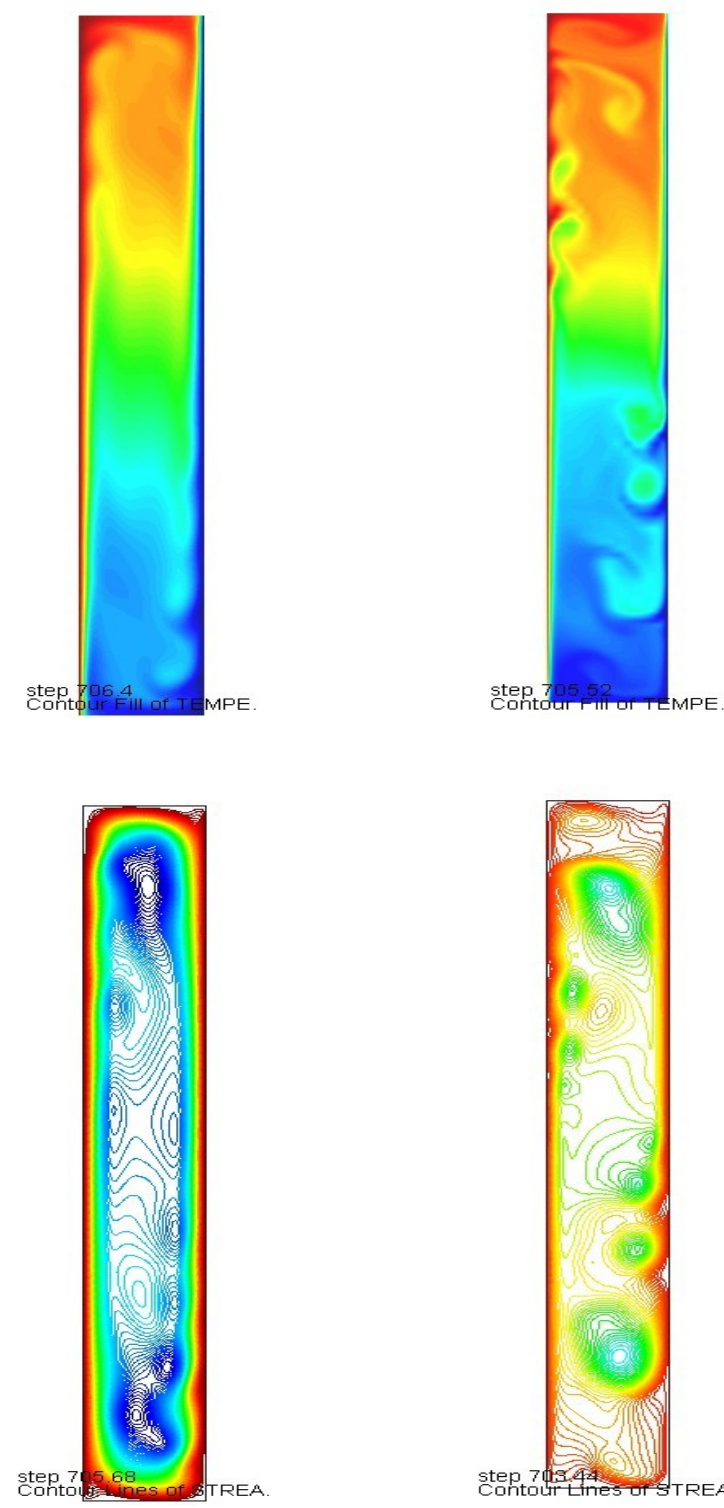

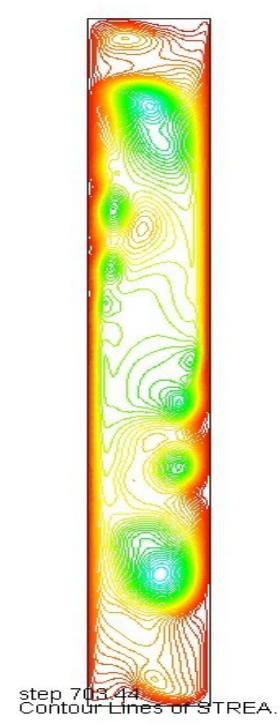

Figure 1: Temperature contours (top) and streamlines (bottom) for the three different Rayleigh numbers: $\mathrm{Ra}=3.4510^{5}, \mathrm{Ra}=10^{6}$ and $\mathrm{Ra}=10^{7}$. 

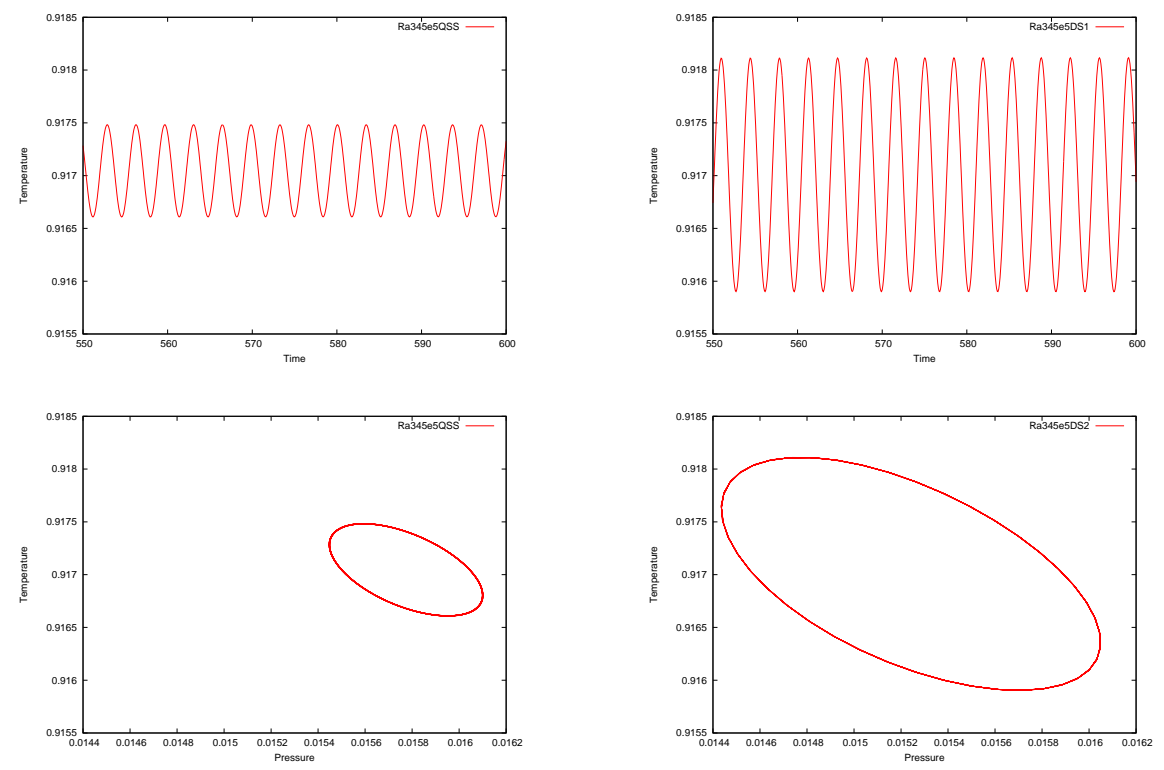

Figure 2: Results for $\mathrm{Ra}=3.4510^{5}$. Left: QSS; right: DS; top: temperature evolution; bottom: pressure-temperature cycle.
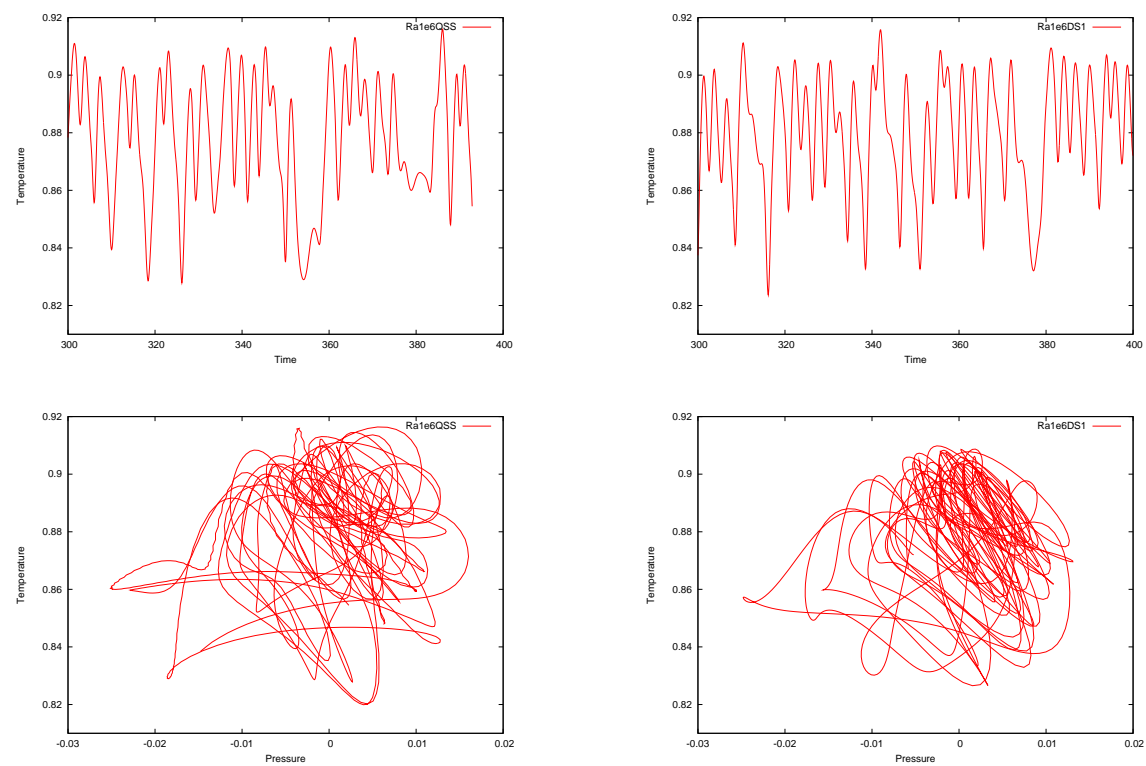

Figure 3: Results for Ra $=10^{6}$. Left: QSS; right: DS; top: temperature evolution; bottom: pressuretemperature cycle.

\section{Numerical examples}

\subsection{Flow in a differentially heated cavity with aspect ratio 8}

As a first example of application of the formulation presented, we have modeled the flow in a differentially heated cavity with aspect ratio 8 . The data of the problem can be found in [5]. The interest of this 

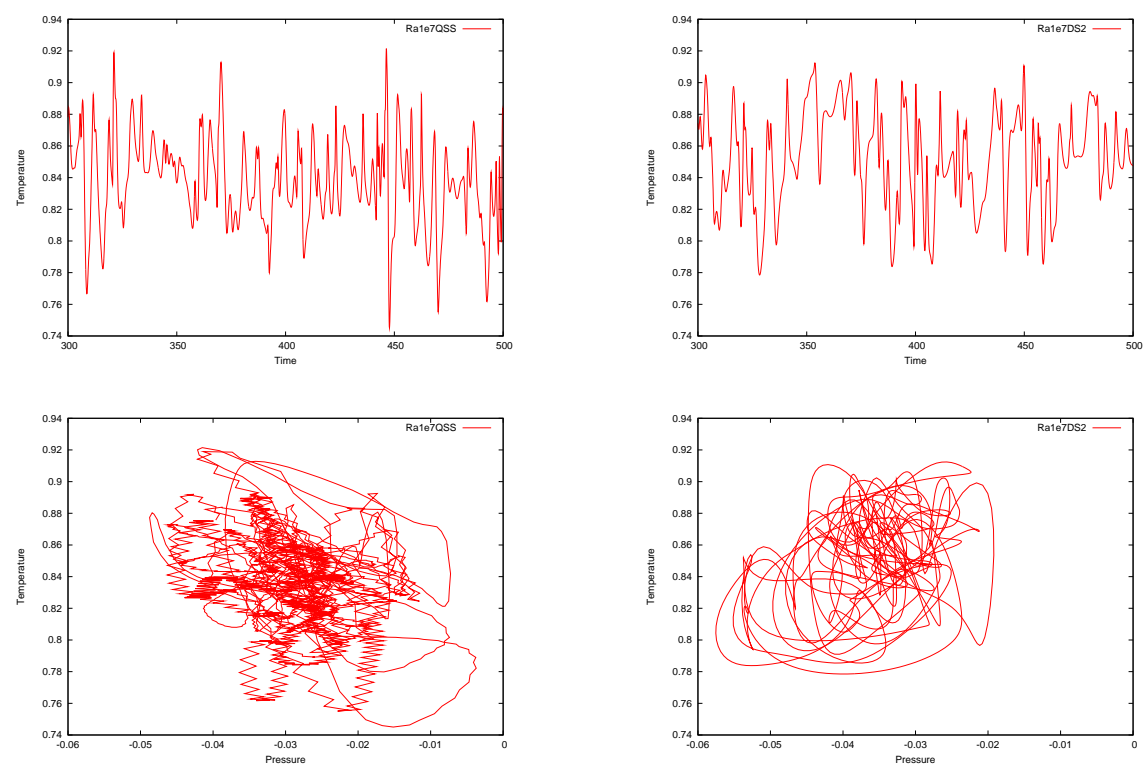

Figure 4: Results for Ra $=10^{7}$. Left: QSS; right: DS; top: temperature evolution; bottom: pressuretemperature cycle.

problem in that it displays transition to chaos as the Rayleigh number is increased.

Three different Rayleigh numbers will be considered: $\mathrm{Ra}=3.4510^{5}$, where it is known that a Hopf bifurcation has occurred and the flow is oscillatory, $\mathrm{Ra}=10^{6}$ and $\mathrm{Ra}=10^{7}$. Chaotic behavior is expected in the last two cases, which is fully developed for the highest Rayleigh number.

In Fig. 1 snapshots of temperature contours and streamlines at a certain time step and for the three Rayleigh numbers are shown, with the only purpose to have an impression of the flow pattern. These and the following results have been obtained on a mesh of 10721 nodal points and 10500 bilinear quadrilateral elements. A second order BDF time integration scheme has been used for the NavierStokes and heat equation, whereas the subscales have been integrated using a backward Euler scheme. The time step size used is 0.08 .

It is not our purpose here to compare the results obtained against others than can be found in the literature, but to see the effect of considering the subscales time-dependent. In this case we will label the resulting formulation DS, for dynamic subscales. When the time derivative of the subscales is neglected and $\boldsymbol{a} \approx \boldsymbol{u}_{h}$ is used as advection velocity, we will label the method as QSS, for quasi-static subscales.

To analyze the dynamical response of the formulation, we have plotted the temperature evolution at the point in the middle of the cavity, as well as the pressure-temperature cycle. Results are shown in Fig. 2 to Fig. 4. The conclusions that may be drawn from these pictures are:

- For Ra $=3.4510^{5}$, both DS and QSS show the expected oscillatory behavior. However, DS has a wider $p-\vartheta$ cycle, indicating less dissipation.

- For Ra $=10^{6}$ results obtained using DS and QSS are very similar. They both display chaos, as it can be observed from the $p-\vartheta$ cycle.

- For $\mathrm{Ra}=10^{7}$ the solution obtained is fully chaotic. A very important point to notice is that QSS has some oscillations in time, particularly visible in the $p-\vartheta$ cycle, that do not appear using DS. We have observed the same behavior in other problems [11,9]. 


\subsection{Flow over a surface mounted obstacle}

In this subsection we present a numerical experiment showing the relationship between the mechanical and the thermal dissipation associated to the numerical model we propose. This example is taken from [23], to where the reader is referred for details of the calculation.

The problem consists in modeling the flow over a surface mounted obstacle, consisting in a cylinder of square cross section. The domain is discretized using a finite element mesh of around 2.2 million linear tetrahedral elements. Just to have a feeling of the flow, the instantaneous velocity contours (at a certain time instant) in the mid section of the channel where the flow takes place are plotted in the top picture of Fig. 5. Velocity boundary conditions are prescribed on the left boundary, whereas zero velocity is fixed on the bottom surface and zero normal velocity on the top boundary of the computational domain. The outflow (right boundary) is left free.

Concerning the setting for the thermal analysis, only the temperature on the obstacle is fixed to 1 , whereas the rest of the boundary is assumed adiabatic. The thermal expansion coefficient is $\alpha=0$ (no Boussinesq coupling) and two Prandtl numbers have been considered, namely, $\operatorname{Pr}=1$ and $\operatorname{Pr}=100$.

The interesting fact of this numerical simulation is to see which are the values obtained for the turbulent Prandtl number associated to the formulation as given by (51) (with $c_{1}=4$ and $c_{2}=2$, the numerical parameters we use in the calculations with linear elements). In Fig. 5 we have plotted the temperature contours at a certain time instant and the turbulent Prandtl number for $\operatorname{Pr}=1$ and $\operatorname{Pr}=100$. The conclusion is clear: since the dissipations in (51) (or the local Péclet number in estimate (53)) change from point to point, so does the turbulent Prandtl number. In Fig. 5 the local Péclet number has been computed with a characteristic velocity per element, and it is therefore constant within each element of the finite element mesh. It is observed that there are many elements in which the turbulent Prandtl number is far from the value $\mathrm{Pr}_{\text {tur }}=1$ usually adopted in LES models.

\section{Conclusions}

The purpose of this paper has been to give a complete overview of a finite element formulation for thermally coupled incompressible whose intention is to go beyond stabilized finite element methods and, more precisely, to allow to simulate turbulent flows. Even though most of the points have been treated succinctly, the main aspects of the method have been touched, namely:

- Its derivation through a scale splitting in the variational multiscale context.

- The definition of the stabilization parameters through an approximate Fourier analysis of the problem from a procedure applicable to general systems of equations.

- The possibility of considering dynamic subscales.

- The choice of the space of subscales as orthogonal to the finite element space.

Relevant to the possibility of simulating thermally coupled turbulent flows, we have analyzed:

- The conservation properties of the formulation in terms of the expression of the convective term.

- The dissipative structure, identifying the energy transfer terms and the possibility to have scale separation and to model backscatter.

- The numerical dissipation for both the Navier-Stokes and the heat equation, introducing a unambiguous numerical definition for the turbulent Prandtl number. 

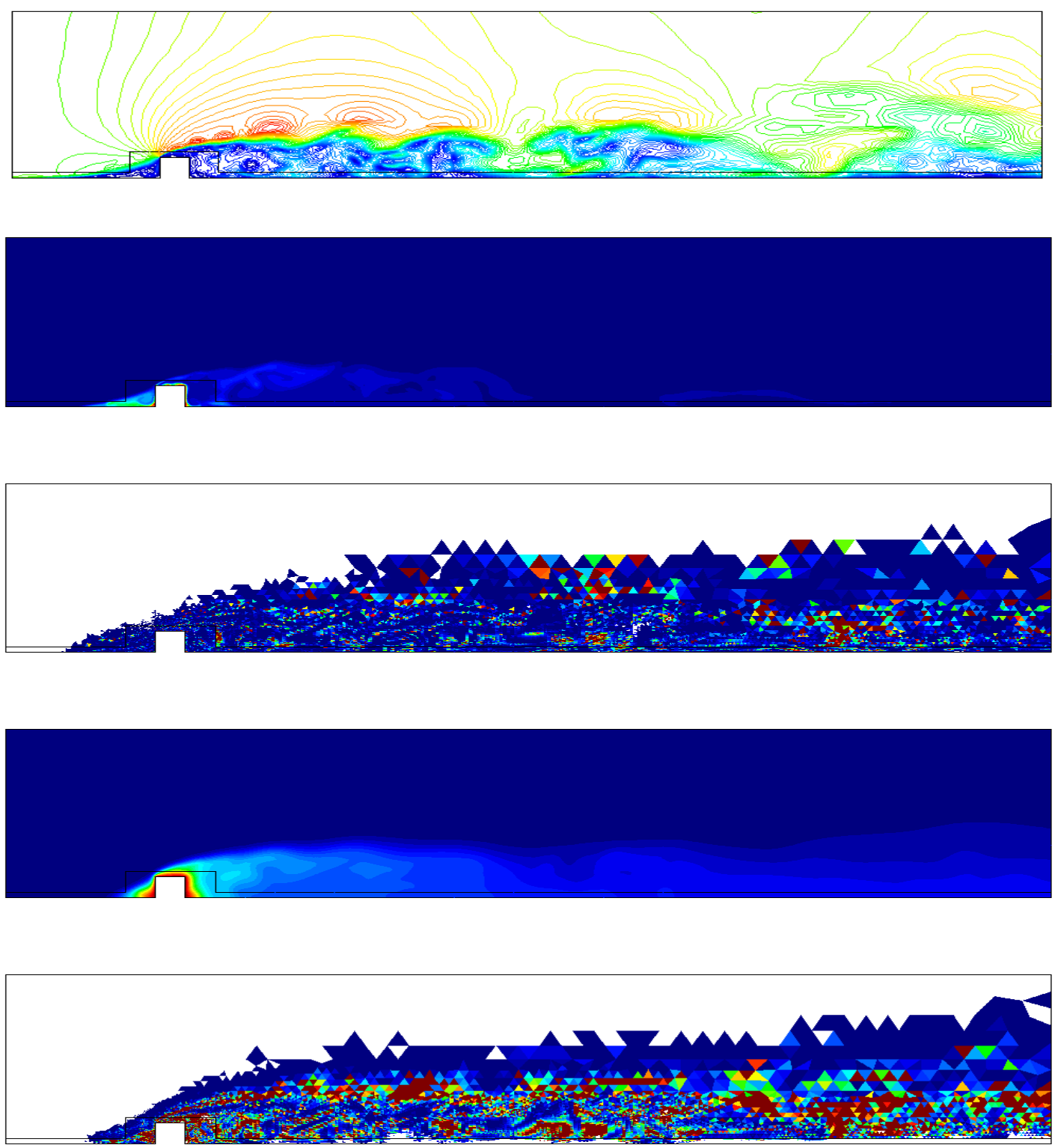

Figure 5: Results for the flow over a surface mounted obstacle in the mid section of the channel (at a certain time instant). From the top to the bottom: (1) Instantaneous velocity contours. (2) Instantaneous temperature contours for $\operatorname{Pr}=1$. Maximum $=1$ (red), minimum $=0$ (blue). (3) Effective turbulent Prandtl number for $\operatorname{Pr}=1$. Cut-off at 10 (red). (4) Instantaneous temperature contours for $\operatorname{Pr}=100$. Maximum $=1$ (red), minimum $=0$ (blue). (5) Effective turbulent Prandtl number for $\operatorname{Pr}=100$. Cut-off at 10 (red). 
Even though there are many questions left open, we believe that the material presented here is a clear indication of the potential of the approach we propose to model turbulence, particularly in the case of thermally coupled flows.

\section{Acknowledgement}

This work has been partially supported by project FITUN, ref. TRA2008-05162, from the Spanish Ministry of Science and Innovation.

\section{References}

[1] S. Badia and R. Codina. On a multiscale approach to the transient Stokes problem. Transient subscales and anisotropic space-time discretization. Applied Mathematics and Computation, 207:415-433, 2009.

[2] Y. Bazilevs, V.M. Calo, J.A. Cottrell, T.J.R. Hughes, A. Reali, and G. Scovazzi. Variational multiscale residual-based turbulence modeling for large eddy simulation of incompressible flows. Computer Methods in Applied Mechanics and Engineering, 197:173-201, 2007.

[3] P.B. Bochev, M.D. Gunzburger, and R.B. Lehoucq. On stabilized finite element methods for the Stokes problem in the small time-step limit. International Journal for Numerical Methods in Fluids, 53:573-597, 2007.

[4] J.P. Boris, F.F. Grinstein, E.S. Oran, and R.L. Kolbe. New insights into large eddy simulation. Fluid Dyn. Res., 10:199-228, 1992.

[5] M.A. Christon, P.M. Gresho, and S.B. Sutton. Computational predictability of natural convection flows in enclosures. International Journal for Numerical Methods in Fluids, 40:953-980, 2002.

[6] R. Codina. Stabilized finite element approximation of transient incompressible flows using orthogonal subscales. Computer Methods in Applied Mechanics and Engineering, 191:4295-4321, 2002 .

[7] R. Codina. Analysis of a stabilized finite element approximation of the Oseen equations using orthogonal subscales. Applied Numerical Mathematics, 58:264-283, 2008.

[8] R. Codina, J.M. González-Ondina, G. Díaz-Hernández, and J. Principe. Finite element approximation of the modified Boussinesq equations using a stabilized formulation. International Journal for Numerical Methods in Fluids, 57:1305-1322, 2008.

[9] R. Codina and J. Principe. Dynamic subscales in the finite element approximation of thermally coupled incompressible flows. International Journal for Numerical Methods in Fluids, 54:707$730,2007$.

[10] R. Codina, J. Principe, and J. Baiges. Subscales on the element boundaries in the variational two-scale finite element method. Computer Methods in Applied Mechanics and Engineering, 198:838-852, 2009.

[11] R. Codina, J. Principe, O. Guasch, and S. Badia. Time dependent subscales in the stabilized finite element approximation of incompressible flow problems. Computer Methods in Applied Mechanics and Engineering, 196:2413-2430, 2007. 
[12] P.A.B. de Sampaio, P.H. Hallak, A.L.G.A. Coutinho, and M.S. Pfeil. A stabilized finite element procedure for turbulent fluid-structure interaction using adaptive time-space refinement. International Journal for Numerical Methods in Fluids, 44:673-693, 2004.

[13] O. Guasch and R. Codina. A heuristic argument for the sole use of numerical stabilization with no physical LES modelling in the simulation of incompressible turbulent flows. Submitted.

[14] J.O. Hinze. Turbulence. McGraw-Hill, 1975.

[15] J. Hoffman and C. Johnson. A new approach to computational turbulence modeling. Computer Methods in Applied Mechanics and Engineering, 195:2865-2880, 2006.

[16] T.J.R. Hughes. Multiscale phenomena: Green's function, the Dirichlet-to-Neumann formulation, subgrid scale models, bubbles and the origins of stabilized formulations. Computer Methods in Applied Mechanics and Engineering, 127:387-401, 1995.

[17] T.J.R. Hughes, G.R. Feijóo, L. Mazzei, and J.B. Quincy. The variational multiscale method-a paradigm for computational mechanics. Computer Methods in Applied Mechanics and Engineering, 166:3-24, 1998.

[18] T.J.R. Hughes, L. Mazzei, and K.E. Jansen. Large eddy simulation and the variational multiscale method. Computing and Visualization in Science, 3:47-59, 2000.

[19] T.J.R. Hughes and G.N. Wells. Conservation properties for the Galerkin and stabilised forms of the advection-diffusion and incompressible Navier-Stokes equations. Computer Methods in Applied Mechanics and Engineering, 194:1141-1159, 2005.

[20] D.K. Lilly. The representation of small-scale turbulence theory in numerical simulation experiments. In H.H. Goldstine, editor, Proc. IBM Scientific Computing Symp. on Environmental Sciences, 1967.

[21] S.B. Pope. Turbulent Flows. Cambridge University Press, 2000.

[22] J. Principe and R. Codina. A stabilized finite element approximation of low speed thermally coupled flows. International Journal of Numerical Methods for Heat \& Fluid Flow, 18:835-867, 2008 .

[23] J. Principe, R. Codina, and F. Henke. The dissipative structure of variational multiscale methods for incompressible flows. Computer Methods in Applied Mechanics and Engineering, To appear.

[24] J.N. Reddy and D.K. Gartling. The finite element method in heat transfer and fluid dynamics. CRC Press, 1994.

[25] P. Sagaut. Large Eddy Simulation for Incompressible Flows. Scientific Computing, Springer, 2001.

[26] F. Shakib and T.J.R. Hughes. A new finite element formulation for computational fluid dynamics: IX. Fourier analysis of space-time Galerkin/least-squares algorithms. Computer Methods in Applied Mechanics and Engineering, 87:35-58, 1991.

[27] T. Tezduyar and S. Sathe. Stabilization parameters in SUPG and PSPG formulations. Journal of Computational and Applied Mechanics, 4:71-88, 2003.

[28] D.C. Wilcox. Turbulence modeling for CFD. DCW Industries, Inc. La Cañada, California, 1993. 